\title{
"A comparative in vitro and in vivo study of osteogenicity by using two biomaterials and two human mesenchymal stem cell subtypes."
}

Author list: Lucile Fievet, $\mathrm{MD}, \mathrm{PhD}^{1^{*}}$; Nicolas Serratrice, $\mathrm{MD}, \mathrm{PhD}^{2,3^{*}}$; Bénédicte Brulin, Eng ${ }^{4}$; Laurent Giraudo, Tech ${ }^{3}$; Julie Véran, $\mathrm{PhD}^{3}$; Nathalie Degardin, $\mathrm{MD}^{5}$; Florence Sabatier, PharmD, $\mathrm{PhD}^{3}$; François Féron, $\mathrm{PhD}^{3,6 \S}$; Pierre Layrolle, $\mathrm{PhD}^{4 \S}$.

1. Department of Pediatric Surgery, Centre Hospitalier Régional Henri Duffaut; Avignon, France.

2. Department of Neurosurgery, La Timone Hospital (AP-HM); Marseille, France.

3. Culture and Cell Therapy Laboratory (AP-HM), CIC BT 1409; Marseille, France.

4. INSERM, UMR 1238, PHY-OS, Bone sarcomas and remodeling of calcified tissues, Faculty of Medicine, Nantes University; Nantes, France.

5. Department of Pediatric Surgery, La Timone Enfant Hospital (AP-HM); Marseille, France.

6. Aix Marseille University, CNRS, INP; Marseille, France.

* These authors contribute equally to the manuscript.

$\S$ Equally supervising authors. 


\section{Corresponding author:}

Lucile Fievet, MD, PhD

Department of Pediatric Surgery, Centre Hospitalier Régional Henri Duffaut

305, rue Raoul Follereau

84000 Avignon, France

lucilefievet@hotmail.com or fievet.lucile@ch-avignon.fr 
Funding Statement: This work was supported by AORC Junior 2014-2015 grant [grant number NB/JM/1144-14, 2015, recipient LF].

Financial Disclosure Statement: Authors have nothing to disclose.

Presented at: the $73^{\text {th }}$ congress of the French Pediatric Surgery Society (SFCP) 2016 Toulouse France

Running Title: Osteogenicity with biomaterials and MSCs

\section{List of abbreviations:}

MSCs = mesenchymal stromal stem cells

hMSCs = human mesenchymal stem cells

$\mathrm{BM}-\mathrm{MSC}=$ bone marrow derived MSCs

NE-MSCs = nasal ecto-mesenchymal stem cells

$\mathrm{GMP}=$ good manufacturing practice

$\mathrm{BCP}=$ biphasic calcium phosphate

HA = hydroxyapatite

$\beta T C P=$ beta-tricalcium phosphate

$B G=$ bioactive glass

PBS $=$ phosphate buffered saline

CPS = calcium phosphate supersaturated solution

PFA = paraformaldehyde

RTqPCR = real time quantitative PCR

GAPDH = glyceraldehyde 3-phosphate dehydrogenase 
$\mathrm{B} 2 \mathrm{M}=$ beta-2-microglobulin

RUNX2 = RUNX family transcription factor 2

$\mathrm{BSP}=$ bone salioprotein

COLA1 = collagen $A 1$

$\mathrm{OC}=$ osteocalcin

BMP2 = bone morphogenic protein 2

BMP4 = bone morphogenic protein 4

ALP = alkaline phosphatase

$\mathrm{OP}=$ osteopontin

$\mathrm{RT}=$ room temperature 


\section{Abstract:}

Bone repair induced by stem cells and biomaterials may represent an alternative to autologous bone grafting. Here, we compared the efficiency of two biomaterials - biphasic calcium phosphate (BCP) and bioactive glass (BG) - when loaded with either adult bone marrow mesenchymal stem cells (BM-MSCs) or newborn nasal ecto-mesenchymal stem cells (NE-MSCs), the latter being collected for further repair of lip cleft-associated bone loss. Both cell types display the typical stem cell surface markers CD73+/CD90+/CD105+/nestin, and exhibit the MSC-associated osteogenic, chondrogenic and adipogenic multipotency. NEMSCs produce less collagen and alkaline phosphatase than BM-MSCs. At the transcript level, NE-MSCs express more abundantly three genes coding for bone sialoprotein, osteocalcin and osteopontin, while BM-MSCs produce extra copies of RUNX2. BM-MSCs and NE-MSCs adhere and survive on $\mathrm{BCP}$ and $\mathrm{BG}$. In vivo experiments reveal that bone formation is only observed with BM-MSCs transplanted on BCP biomaterial. 


\section{Introduction:}

Biomaterial- and stem cell-associated bone repair represents a very promising alternative to autologous bone grafting. The most commonly investigated cells are mesenchymal stem cells (MSCs) ${ }^{1-4}$, among them, bone marrow derived MSCs (BM-MSCs) tend to be considered as the gold standard due to their capacity in combination with biomaterial scaffolds to repair bones in vivo ${ }^{5-7}$. However, other sources of MSCs have been poorly investigated ${ }^{8}$. Our laboratory identified and purified from the lamina propria of the human olfactory mucosa, the ecto-mesenchymal stem cells, originate from the neural crest, that reside in the craniofacial area during adulthood ${ }^{9-11}$. When compared to BM-MSCs, nasal ecto-mesenchymal stem cells (NE-MSCs) display an inclination to differentiate into osteocytes, rather than chondrocytes or adipocytes, and produce mineralized bone tissue in vivo after being filled on calcium phosphate ceramic discs when implanted subcutaneously in nude mice ${ }^{12}$.

Many biomaterials have been developed to offer surgeons and patients safe alternatives to autologous bone graft, that is limited in quantity and adds morbidity at the harvesting site. The most commonly used are composed of biphasic calcium phosphate (BCP), a mixture of hydroxyapatite $(\mathrm{HA})$ /beta-tricalcium phosphate $(\beta T C P)$, similar to the mineral bone composition, but they display an insufficient osteoinductive capacity to regenerate large bone defects. BM-MSCs added to porous calcium phosphate ceramics induced bone formation when implanted under the skin of nude mice ${ }^{6,13-15}$, in rat and pig femurs ${ }^{16,17}$. This BCP/BMMSCs combination has even demonstrated safe bone regeneration in clinical trials ${ }^{18-20}$. Most recently, a synthetic bioactive glass (BG) or bioglass (bioglass $45 \mathrm{~S} 5$ or GlassBONE ${ }^{\mathrm{TM}}$ ) has also been used for bone regeneration in orthopedic, traumatic, spinal and craniomaxillo-facial surgery. Based on borate and borosilicate, BG enhances bone and underlying neocartilage formation when compared to silicate bioactive glass ${ }^{\mathbf{2 1}, 22}$. Moreover, borate-based BG displays 
controllable degradation rates, and can be doped with trace quantities of elements such as $\mathrm{Cu}, \mathrm{Zn}$ and $\mathrm{Sr}$, which are known to be beneficial for healthy bone growth. In addition, it has been observed that BG promotes angiogenesis, which is critical in bone tissue regeneration ${ }^{23}$. In order to go further, we design a new study comparing the osteogenic capacity of human mesenchymal stem cells (hMSCs), derived from either adult bone marrow or newborn nasal cavity (having in the mind the repair of cleft lips), associated with BCP or BG biomaterials, in vitro and after subcutis implantation in nude mice. 


\section{Results:}

\section{Newborn nasal stem cells display an NE-MSC phenotype}

Human newborn nasal cells being cultivated for the first time in the team, we first assessed their stemness. They express nestin (Figure 1A), a widely recognized marker for stem cells. In addition, they produce CD73, CD90 and CD105 (Figures 1B-D), as documented for adult NEMSCs, and not CD45, a specific hematopoietic stem cell marker (Figure 1E).

\section{In vitro assessment of stem cell multipotency}

We compared the capacity of both MSC types to differentiate in vitro into osteocytes, chondrocytes, and adipocytes, using the usual stains: alizarin red, alcian blue, and red oil (Figure 2A-C). BM-MSCs and NE-MSCs display an intense mineralization at D14, and exhibit a similar pattern of differentiation for chondrocytes and adipocytes.

\section{BM-MSCs and NE-MSCs adhere and survive on biomaterials}

Both BM-MSCs and NE-MSCs adhere to BCP and BG (Figure 3A). BG, and not BCP, induces cell death (Figure 3B), as early as D2 for BM-MSCs, and only at D21 for NE-MSCs, a possible indication that stem cells from human newborns are more resistant to a relatively adverse material.

\section{BM-MSCs display a higher proliferation on BG biomaterial}

On plastic, BM-MSCs and NE-MSCs display a high proliferation rate, although the latter overcomes the former ${ }^{12}$. It was then questionable to confirm this finding when using biomaterials. Figure 4 indicates that, on both biomaterials, NE-MSCs proliferate more rapidly than BM-MSCs at the start of the experiment. However, at D14, the numbers are even and, 
subsequently, due to cell death occurring at this stage (see above), the density of both MSCs declines (Figures 4A-B). Figures 4C-E indicates that, when compared to BCP, BG favors BMMSC proliferation. No difference between the two materials observed for NE-MSCs.

\section{Collagen production increases in BM-MSCs and decreases in NE-MSCS}

BM-MSCs produce more collagen, being a chondrocytic and osteogenic specific marker (Sirius red staining), than NE-MSCs. Noticeably, collagen expression timely increases in BM-MSCs, and declines in NE-MSCs (Figures 5A).

\section{BM-MSCs produce ALP only on BG biomaterial}

BM-MSCs and NE-MSCs produced ALP on plastic (Figure 5B). At D7, BM-MSCs express ALP only when cultivated on BG, while NE-MSCs produce ALP on both biomaterials with a higher production in proliferative medium (Figure $\mathbf{5 C}$ ). To move further and quantify ALP production in various conditions, we assessed its expression in accordance with cell abundance (Figure 6A-B). NE-MSCs express an important amount of ALP when grown on BG in osteogenic conditions, and conversely is negligible on BCP, except at D1 (Figure 6C).

\section{A differentiated expression of osteocyte-associated genes}

Discrepancies between both MSCs further analyzed by measuring the expression of 8 boneassociated genes at D1 and D7 on both biomaterials (Figure 7 and Supplementary Figure 4). Normalization of gene expression performed using the amount expressed by BM-MSCs at D1 on $B C P$, as basal level. When cultivated on BG, NE-MSCs overexpress the genes coding for BSP, $O C$ and $O P$ (Figures 7A-C). Conversely, on the same biomaterial, BM-MSCs produce a higher number of transcripts coding for RUNX2 (Figure 7D). No significant difference between the 
two groups noted for other genes (Figures 8A, 8C, 8D). ALP gene expression exhibited quite similar variations to ALP in culture supernatant, except for BCP at D1 (Figure 8B). No data are provided for BCP at D7 because a very small amount of RNA was extracted. This may be due to the failure of cell adhesion and proliferation on $\mathrm{BCP}$, which can also explain the absence of correlation between ALP quantification and ALP gene expression at D1 on BCP. Indeed, reproducibility of experiment was affected by the absence of pre-incubation of $\mathrm{BCP}$ in culture medium prior to cell seeding.

\section{In vivo experiments}

8 weeks after subcutaneous implantation in nude mice, none of the cell-free BG was found to harbor bone cells and vascularized fibrous tissue (Figure 9A). No bone formation was either observed on the same biomaterial loaded with BM-MSCs or NE-MSCs (Figure 9C). BM-MSCs gave rise to bone tissue when grafted on BCP (Figure 9A-B). Conversely, NE-MSCs failed to produce osteocytes (Figures 9A-B). No signs of systemic or local toxicity, no infections, and no behavioral changes were observed with both biomaterials with or without MSCs. 


\section{Discussion:}

In this study, we compared the in vivo osteoinduction potential of hMSCs derived from either bone marrow or child nose associated with BCP or BG, two synthetic bone fillers currently used for their favorable bioactivity and osteoconductive properties ${ }^{7,18,27}$. Alone these biomaterials have insufficient osteoinductive properties to regenerate large bone defects. Combinations of hMSCs and BCP have been shown to induce de novo bone in ectopic sites and thus, have been widely studied in vivo as alternatives to autologous bone grafts ${ }^{6,20}$. On the opposite, combinations of MSCs and BG have been mainly studied in vitro,but poorly in vivo ${ }^{\mathbf{2 4}}$. Although belonging to the same superfamily, BM-MSCs and NE-MSCs exhibit striking differences, in vitro and in vivo. For future clinical applications, the association of BM-MSCs with $\mathrm{BCP}$ biomaterial seems to be the most promising. Indeed, our in vivo experiments reveal that osteoinduction is only observed when BM-MSCs transplanted on BCP. Recently, Rodrigues and colleagues reported that allogenic adipose-derived MSCs associated with BG is i) biocompatible in the long term (3 months), ii) maintain their osteoinduction potential, and iii) is safe after subcutaneous implantation in immunocompetent balb-c mice. Indeed, a low spreading during cell adhesion was detected associated with an increase of HA depositions around the cells that looks like differentiated osteocytes. Any apparent local or systemic toxicity for organs, or strong immunogenic reactions were noted, except a vascularized dense capsule around the graft ${ }^{28}$.

Certain in vivo conditions could significatively impact the engraftment and the bone formation. In our study we chose to use nude mice to limit the immune response against the MSC allograft. The MSC allograft in Balb-c mice is possible, but the capsule formation surrounding the graft, probably due to the immune response in immunocompetent mice, could be a complication for implants ${ }^{28-30}$. The $\mathrm{pH}$ and the calcium concentration can also affect 
the cells. Here, we worked with $4 \mathrm{mM}$ of calcium in culture medium. Maeno et al. showed that high concentration of calcium above $10 \mathrm{mM}$ are cytotoxic to osteoblasts, but those below $8 \mathrm{mmol}$ promote cell proliferation ${ }^{31}$. We also used medium supplemented with $5 \%$ GMPgrade human PL for MSC culture. It is now well established that animal derived products as FCS could significantly interact with phenotypical and functional characteristics of BM$\mathrm{MSCs}^{25,26}$. Here, MSCs were grown with biomaterials $24 \mathrm{~h}$ before graft, and we implanted $2 \times 10^{6} \mathrm{MSC}$ per site. In comparison, Rodrigues et al. used $2 \times 10^{4} \mathrm{MSC}$ grown during $48 \mathrm{~h}$ on BG before implantation ${ }^{28}$. 


\section{Materials and Methods:}

\section{Biomaterials}

Two biomaterial scaffolds for hMSCs compared: macro/microporous BCP granules $0.5-1 \mathrm{~mm}$ in size $\left(\mathrm{MBCP}+{ }^{\circledR} \text {, Biomatlante, Vigneux de Betagne, France }\right)^{6,20}$, and $\mathrm{BG}$ granules $0.5-1 \mathrm{~mm}$ in size (GlassBONE ${ }^{T M}$, Noraker, Villeurbanne, France) ${ }^{24}$. BCP is a ceramic composed of HA/ $\beta T C P$ in a ratio of $20 / 80$ by weight. $\mathrm{BG} 45 \mathrm{~S} 5$ composed of $45 \mathrm{wt} . \% \mathrm{SiO}_{2}, 24.5$ wt. $\% \mathrm{Na}^{2} \mathrm{O}$ and 24.5 wt.\% $\mathrm{CaO}$.

\section{Donors}

Bone marrow aspirates obtained from the iliac crest by standard puncture and aspiration of healthy human donors ( 21 and 26 years old), after receiving informed consent according to the Declaration of Helsinki. The project approved by the Ethical Committee of ULM University. BM-MSCs used in this study are of GMP grade, and expanded according to previously published protocols ${ }^{6}$. In brief, BM-MSCs isolated from heparinized bone marrow aspirates by seeding 50,000 white blood cells/ $\mathrm{cm}^{2}$ on two-chamber CellStacks (Corning/VWR, Ulm, Germany) in $\alpha$-MEM (Lonza, Basel, Switzerland) supplemented with 5\% GMP-grade human platelet lysate (PL) (IKT, Ulm, Germany) in order to avoid animal products ${ }^{25,26}$. Cells cultured for 10 or 14 days with medium exchange twice per week. Cells detached and reseeded at a density of 4,000 BM-MSCs/cm² on two-chamber Cellstacks in $\alpha$-MEM supplemented with $8 \%$ PL for a further 5 or 7 days.

Newborn NE-MSCs purified from the nasal mucosa, collected during a scheduled intervention for closing a cleft palate, under general anaesthesia. The procedure approved by an ethical committee (CPP, RCB: 2015-A00984-45). Two infants included in this pilot study. Biopsies sliced in small pieces, and incubated in $1.5 \mathrm{~mL}$ of collagenase (NB5, $1 \mathrm{U} / \mathrm{mL}$, Nordmark 
Biochemicals) for $60 \mathrm{~min}$ at $37^{\circ} \mathrm{C}$, before being mechanically dissociated. After digestion, $7 \mathrm{~mL}$ of serum containing culture medium added. After centrifugation, the cell pellet resuspended in DMEM/HAM supplemented with antibiotics (penicillin/gentamicin and fungizone), and Gibco $^{\text {TM }}$ fetal calf serum (FCS) (Thermo Fisher Scientific, Waltham, Massachusetts, USA)on $25 \mathrm{~cm}^{2}$ plates. After 10 days, cells replated at a density of 4,000 cells $/ \mathrm{cm}^{2}$ on $175 \mathrm{~cm}^{2}$ plates. At confluency, cells detached with Gibco ${ }^{\mathrm{TM}}$ trypsin EDTA 0.05\% (Thermo Fisher), and NE-MSCs stored in liquid nitrogen. Banking for scientific research performed anonymously, according to the rules of the Ministry of Higher Education and Research (number NDC-2011-1331).

\section{Bioactive Glass pre-incubation}

BG granules incubated in $1 \mathrm{ml}$ of phosphate buffered saline (PBS) for $5 \mathrm{~h}$, leading to a highly basic $\mathrm{pH}$ 10. In order to get a neutral $\mathrm{pH}$, the BG granules (90 $\mathrm{mg}$ ) incubated overnight in $1 \mathrm{~mL}$ of a calcium phosphate supersaturated solution (CPS). CPS contained $4 \mathrm{mM} \mathrm{CaCl} 2.2 \mathrm{H}_{2} \mathrm{O}$, and $2 \mathrm{mM}$ of $\mathrm{Na}_{2} \mathrm{HPO}_{4} .2 \mathrm{H}_{2} \mathrm{O}$ in $0.9 \% \mathrm{NaCl}$ buffered at $\mathrm{pH} 7.4$ with TRIS/HCl. After an overnight soaking in CPS, the $\mathrm{pH}$ of supernatant remained at 9. Based on these results, it was decided to seed the cells directly on the biomaterials without pre-incubation.

\section{Culture of MSCs with biomaterials}

Culture of human BM-MSCs and NE-MSCs with biomaterials performed in Corning ${ }^{\circledR}$ Costar $^{\circledR}$ ultra-low attachment 24 well plates (Merck KGaA, Darmstadt, Germany) with Gibco ${ }^{\mathrm{TM}} \alpha-$ MEM (Thermo Fisher), supplemented with $8 \%$ human PL and $1 \%$ penicillin/streptomycin for 2-3 weeks (50 mg BCP or 90 mg BG per well).

\section{Adhesion of MSCS on biomaterials}


Adhesion assessed using TM3000 scanning electron microscope (Hitachi, Krefeld, Germany), operating at an acceleration voltage of $5 \mathrm{kV}$, and imaged at a magnification of $50 \mathrm{x}-500 \mathrm{x}$, after fixation and dehydration of the cells in ethanol.

\section{Viability and proliferation of MSCs}

Cell viability on biomaterials evaluated at D2/7/14/21 by staining live cells with the fluorescent green stain calcein $(1.25 \mu \mathrm{L} / \mathrm{mL})$, and dead cells with fluorescent red ethidium homodimer$1(1 \mu \mathrm{L} / \mathrm{mL})$, according Invitrogen ${ }^{\mathrm{TM}} \mathrm{L}-3224$ kit (Thermo Fisher). Cell proliferation assessed for 21 days using alamarBlue ${ }^{\circledR}$ assay (Thermo Fisher).

\section{hMSC characterization by flow cytometry}

Cells stained with CD90-FITC, CD73-PE, CD105-PC7, CD45-APC-A750 antibodies (Beckman Coulter, Brea, California, USA), or corresponding isotype controls in matched concentration, 20 min at room temperature (RT) and protected from light. Cells washed in PBS without $\mathrm{Ca}^{2+}$ $\mathrm{Mg}^{2+}$ (Thermo Fisher), fixed, and permeabilized (IntraPrep Permeabilization Kit, Beckman Coulter). Intracellular staining then performed by indirect immunofluorescence method with an adapted dilution of Nestin antibody (Merck Millipore, Burlington, Massachusetts, USA), or its corresponding isotype control in matched concentration, and with an Alexa Fluor 647 goat anti-mouse IgG $(\mathrm{H}+\mathrm{L})$ secondary antibody (Thermo Fisher). After washing, cells analyzed with a NAVIOS flow cytometer (Beckman Coulter), and data files interprated using Kaluza software (Beckman Coulter). 
Osteogenic differentiation: both BM-MSCs and NE-MSCs plated at the density of $5 \times 10^{3}$ cells $/ \mathrm{cm}^{2}$ in 24 well plates in basal media. After 1 day, MSC differentiation towards an osteogenic lineage induced with standard osteogenic supplements (10 mM 6 -glycerolphosphate, $250 \mu \mathrm{M}$ ascorbic acid, and $100 \mathrm{nM}$ dexamethasone). At D7/14/21, cells fixed with $4 \%$ paraformaldehyde (PFA), and mineralization detected by staining with a $40 \mathrm{mM}$ alizarin red solution $(\mathrm{pH}$ 4.1-4.3).

Adipogenic differentiation: MSCs plated at the density of $2 \times 10^{4}$ cells $/ \mathrm{cm}^{2}$ in 24 well plates. Cells cultured until reaching $80 \%$ confluency in basal media and then induced towards adipogenic lineage with the StemPro ${ }^{\mathrm{TM}}$ Adipogenesis Differentiation kit (Thermo Fisher). After 14 or 21 days, cells fixed with 4\% PFA, and adipocytes stained with Oil Red O solution in 2propanol diluted to $60 \%$ using deionized water.

Chondrogenic differentiation: collected MSCs were loaded into $15 \mathrm{~mL}$ tubes $\left(5 \times 10^{5}\right.$ cells/tube) in fresh basal media and centrifuged at 1,500 rpm during $5 \mathrm{~min}$. After $24 \mathrm{~h}$, the basal media removed, and StemPro ${ }^{\top M}$ Chondrogenesis Differentiation kit (Thermo Fisher) added to the cell pellet. At D14 or D21, cell pellets fixed in 4\% PFA, and embedded in paraffin. Fixed pellets then cut with a microtome and stained with Alcian blue (Merck KGaA).

Collagen production: visualized at D7/14/21 with Sirius red staining. Cells fixed with 4\% PFA in PBS for 20 min at RT, then washed two times in PBS, and stained with $1 \mathrm{~mL}$ of $1 \mathrm{mg} / \mathrm{mL}$ of Direct red solution (Merck KGaA) in saturated aqueous solution of picric acid (Merck KGaA) for $1 \mathrm{~h}$ at RT. 
Extracellular alkaline phosphate (ALP) qualitatively evaluated at D7/14/21 with Fast-Violet B Salt and Naphthol-AS-MX ALP staining (Merck KGaA). Cells fixed with a solution, containing two volumes of citric acid-sodium citrate $(1.5 \mathrm{~mol} / \mathrm{L})$, and three volumes of acetone for $30 \mathrm{~s}$ at RT. Cells rinsed with deionized water and incubated with a staining solution for $30 \mathrm{~min}$ in the dark. $50 \mathrm{~mL}$ of staining solution contained $48 \mathrm{~mL}$ of distilled water, $12 \mathrm{mg}$ of Fast-Violet $\mathrm{B}$ Salt (F1631, Merck KGaA), and 2 mL of Naphthol-AS-MX ALP solution (855, Merck KGaA).

\section{ALP quantification}

At D1 and D7, MSCs were lysed using 0.1\% Triton $\mathrm{x}-100,5 \mathrm{mM}$ Tris-HCL pH8 solution. After, three freeze/thaw cycles, the amount of double stranded DNA measured in the supernatants using a fluorescent Quant-iT Picogreen dsDNA Assay kit (Thermo Fisher), and the amount of ALP measured using SigmaFast ${ }^{\mathrm{TM}}$ p-Nitrophenyl phosphate (pNPP) tablets (Merck KGaA). A standard curve drawn with serial dilution of PNPP and a known quantity of ALP from bovine intestinal mucosa (Merck KGaA). Then, a known amount of pNPP added to each sample, prior to incubation during $30 \mathrm{~min}$ at $37^{\circ} \mathrm{C}$. The amount of product ( $p$-nitrophenol) determined by reading the absorbance at $405 \mathrm{~nm}$ on a microplate reader, and the amount of ALP quantified using the following equation:

$A L P(U / m L)=A / V / T$ were $A=p N P P$ in $\mu$ mol, $V=$ volume of sample in $\mathrm{mL}, T=$ time of incubation in minutes.

\section{Osteoblastic differentiation by real time quantitative PCR (RTqPCR)}

Total RNA extracted from cultivated cells with DirectZolTM RNA MiniPrep (Zymo Research, Irvine, California, USA). The RNA samples ( $1 \mu \mathrm{g}$ each) reverse transcribed with the maxima $\mathrm{H}$ minus First Strand cDNA Synthesis kit (Thermo Fisher) with oligo-dT primers in a final volume 
of $20 \mu \mathrm{L}$. The expression of each target gene normalized with glyceraldehyde 3-phosphate dehydrogenase (GAPDH) and beta-2-microglobulin (B2M). The 2- $\Delta \Delta$ Ctmethod used to calculate relative expression levels. The relative gene expression normalized at D1 on plastic. Then the expression of the different bone coding genes assessed by RTqPCR .

\section{Primers}

RUNX Family Transcription Factor 2 (human RUNX2/F: gcctaggcgcatttcaga; human RUNX2/R: gctcttcttactgagagtggaagg), Bone sialoprotein (human BSP/F: caatctgtgccactcactgc ; human BSP /R: cagtcttcatttggtgattgc), Collagen Type I Alpha 1 Chain (human COL1A1/F: ctggacctaaaggtgctgct; human COL1A1/R: gctccagcctctccatcttt), Osteocalcin (human OC/F: tgagagccctcacactcctc; human OC/R: ctggagaggagcagaactgg), Bone morphogenetic protein 2 (human BMP2/F: gttcggcctgaaacagagac; human BMP2/R: ccaacctggtgtccaaaagt), Bone morphogenetic protein 4 (human BMP4/F: tcaagattggctgtcaagaatgatg; human BMP4 /R: caggtatcaaactagcatggctcg), Alkaline phosphatase ( human ALP/F: aacaccacccaggggaac; human ALP/R: ggtcacaatgcccacagatt), and Osteogenic protein (human OP/F:gagggcttggttgtcagc; human OP/R: caattctcatggtagtgagttttcc).

\section{In vivo experiments}

The in vivo experiment conducted according to the European regulation (Directive 2010/63/UE). The approval of the study obtained from the local ethical committee on animal experiment (number6575). Eighteen 7 week-old female nude mice used (NMRI nu/nu, Janvier Labs). 6 groups considered: BCP; BCP/BM-MSCs; BCP/NE-MSCs; BG; BG/BM-MSCs; BG/NE- 
MSCs on $50 \mathrm{mg}$ of BCP or $90 \mathrm{mg}$ of BG. No pre-treatment performed with BG granules. $2 \times 10^{6}$ cells seeded on biomaterial granules and incubated overnight at $37^{\circ} \mathrm{C}$ with $5 \% \mathrm{CO}_{2}$ prior to implantation. Two identical subcutaneous implantation per mouse performed. For statistics, 6 implants per group considered. Animals operated under general anaesthesia with Isoflurane (Abbvie, North Chicago, Illinois, USA). A centimetric skin incision performed on each side of mouse spine. Subcutaneous pockets filled with granules, embedded with or without MSCs. Wounds closed with non-resorbable suture 4.0 Filapeau (Péters Surgicals, Bobigny, France). Animals controlled every day post-surgery, to monitor the healing of the skin, and anomalies in behaviour. 8 weeks post-implantation, animals were euthanized, and implants fixed in 4\% PFA.

\section{Histology and histomorphometry}

BCP and BG samples dehydrated and embedded in poly-methyl-methacrylate resin (Merck KGaA). Sections performed with Leica SP1600 microtome (Wetzlar, Germany), and bone formation visualized by SEM. BCP samples decalcified in Decalc (Microm Microtech, Brignais, France), dehydrated in ascending series of alcohol, and embedded in paraffin. $4 \mu \mathrm{m}$-thin sections cut with a microtome in the middle of the implant. Sections stained by Masson trichrome in order to visualise newly formed bone tissue, granules, fibrosis, vascularization, and cells. Toxicity and biodegradation of biomaterials also determined.

\section{Statistics}

Data processing and statistical analyses were performed with XLSTAT software (Microsoft, Redmond, Washington, USA). P-values less than 0.05 were considered significant. The results were expressed as mean +/- standard deviation (SD). 


\section{References:}

1. Logeart-Avramoglou D, Anagnostou F, Bizios R, Petite H. "Engineering bone: challenges and obstacles." J Cell Mol Med. 2005;9(1):72-84.

2. Viateau V, Guillemin G, Calando Y, et al. "Induction of a barrier membrane to facilitate reconstruction of massive segmental diaphyseal bone defects: an ovine model." Vet Surg. 2006;35(5):445-52.

3. Viateau V, Guillemin G, Bousson V et al. "Long-bone critical-size defects treated with tissue-engineered grafts: a study on sheep." J Orthop Res. 2007;25(6):741-9.

4. Petite H, Viateau V, Bensaïd W et al. "Tissue-engineered bone regeneration. Nat Biotechnol." 2000;18(9):959-63.

5. Pittenger MF, Mackay AM, Beck SC et al. "Multilineage potential of adult human mesenchymal stem cells." Science. 1999;284(5411):143-7.

6. Brennan MÁ, Renaud A, Amiaud J et al. "Pre-clinical studies of bone regeneration with human bone marrow stromal cells and biphasic calcium phosphate." Stem Cell Res Ther. 2014;5(5):114.

7. Stanovici J, Le Nail LR, Brennan MA et al. "Bone regeneration strategies with bone marrow stromal cells in orthopaedic surgery." Curr Res Transl Med. 2016;64(2):83-90.

8. Brennan MA, Renaud A, Guilloton F et al. "Inferior In Vivo Osteogenesis and Superior Angiogenesis of Human Adipose-Derived Stem Cells Compared with Bone MarrowDerived Stem Cells Cultured in Xeno-Free Conditions." Stem Cells Transl Med. 2017;6(12):2160-2172. Erratum in: Stem Cells Transl Med. 2018;7(3):315.

9. Kaltschmidt B, Kaltschmidt C, Widera D. "Adult craniofacial stem cells: sources and relation to the neural crest." Stem Cell Rev Rep. 2012;8(3):658-71. 
10. Murrell W, Féron F, Wetzig A et al. "Multipotent stem cells from adult olfactory mucosa." Dev Dyn. 2005;233(2):496-515.

11. Girard SD, Devéze A, Nivet E, Gepner B, Roman FS, Féron F. "Isolating nasal olfactory stem cells from rodents or humans. " J Vis Exp. 2011;(54). pii: 2762.

12. Delorme B, Nivet E, Gaillard J et al. "The human nose harbors a niche of olfactory ectomesenchymal stem cells displaying neurogenic and osteogenic properties." Stem Cells Dev. 2010;19(6):853-66.

13. Mankani MH, Kuznetsov SA, Fowler B, Kingman A, Robey PG. "In vivo bone formation by human bone marrow stromal cells: effect of carrier particle size and shape." Biotechnol Bioeng. 2001;72(1):96-107.

14. Arinzeh TL, Tran T, Mcalary J, Daculsi G. "A comparative study of biphasic calcium phosphate ceramics for human mesenchymal stem-cell-induced bone formation." Biomaterials. 2005;26(17):3631-8.

15. Mankani MH, Kuznetsov SA, Robey PG. "Formation of hematopoietic territories and bone by transplanted human bone marrow stromal cells requires a critical cell density." Exp Hematol. 2007;35(6):995-1004.

16. Bruder SP, Kurth AA, Shea M, Hayes WC, Jaiswal N, Kadiyala S. "Bone regeneration by implantation of purified, culture-expanded human mesenchymal stem cells." J Orthop Res. 1998;16(2):155-62.

17. Lebouvier A, Poignard A, Cavet $M$ et al. "Development of a simple procedure for the treatment of femoral head osteonecrosis with intra-osseous injection of bone marrow mesenchymal stromal cells: study of their biodistribution in the early time points after injection." Stem Cell Res Ther. 2015;6:68. 
18. Gjerde C, Mustafa K, Hellem S et al. "Cell therapy induced regeneration of severely atrophied mandibular bone in a clinical trial." Stem Cell Res Ther. 2018;9(1):213.

19. Gómez-Barrena E, Rosset P, Gebhard F et al. "Feasibility and safety of treating nonunions in tibia, femur and humerus with autologous, expanded, bone marrow-derived mesenchymal stromal cells associated with biphasic calcium phosphate biomaterials in a multicentric, non-comparative trial." Biomaterials. 2019;196:100-108.

20. Gamblin AL, Brennan MA, Renaud A et al. "Bone tissue formation with human mesenchymal stem cells and biphasic calcium phosphate ceramics: the local implication of osteoclasts and macrophages." Biomaterials. 2014;35(36):9660-7.

21. Rahaman MN, Day DE, Bal BS et al. "Bioactive glass in tissue engineering." Acta Biomater. 2011;7(6):2355-73.

22. Jones JR. "Review of bioactive glass: from Hench to hybrids." Acta Biomater. $2013 ; 9(1): 4457-86$.

23. Kargozar S, Baino F, Hamzehlou S, Hill RG, Mozafari M. "Bioactive Glasses: Sprouting Angiogenesis in Tissue Engineering." Trends Biotechnol. 2018;36(4):430-444.

24. Rizwan M, Hamdi M, Basirun WJ. "Bioglass ${ }^{\circledR} 45$ S5-based composites for bone tissue engineering and functional applications." J Biomed Mater Res A. 2017;105(11):31973223.

25. Ben Azouna N, Jenhani F, Regaya Z et al. "Phenotypical and functional characteristics of mesenchymal stem cells from bone marrow: comparison of culture using different media supplemented with human platelet lysate or fetal bovine serum." Stem Cell Res Ther. 2012;3(1):6. 
26. Leotot J, Coquelin L, Bodivit G et al. "Platelet lysate coating on scaffolds directly and indirectly enhances cell migration, improving bone and blood vessel formation." Acta Biomater. 2013;9(5):6630-40.

27. Ebrahimi M, Botelho MG, Dorozhkin SV. "Biphasic calcium phosphates bioceramics (HA/TCP): Concept, physicochemical properties and the impact of standardization of study protocols in biomaterials research." Mater Sci Eng C Mater Biol Appl. 2017;71:1293-1312.

28. Rodrigues C, Naasani LIS, Zanatelli C et al. "Bioglass 45S5: Structural characterization of short range order and analysis of biocompatibility with adipose-derived mesenchymal stromal cells in vitro and in vivo." Mater Sci Eng C Mater Biol Appl. 2019;103:109781.

29. Velnar T, Bunc G, Klobucar R, Gradisnik L. "Biomaterials and host versus graft response: a short review." Bosn J Basic Med Sci. 2016;16(2):82-90.

30. Chung L, Maestas DR Jr, Housseau F, Elisseeff JH. "Key players in the immune response to biomaterial scaffolds for regenerative medicine." Adv Drug Deliv Rev. 2017;114:184-192.

31. Maeno S, Niki Y, Matsumoto $\mathrm{H}$ et al. "The effect of calcium ion concentration on osteoblast viability, proliferation and differentiation in monolayer and 3D culture." Biomaterials. 2005;26(23):4847-55. 


\section{Figure legends:}

Figure 1: Characterization of newborn nasal stem cells. Phenotyping of cultivated cells was performed using flow cytometry and stem cell-specific markers. Purified nasal cells were positive for nestin (A), CD73 (B), CD90 (C) and CD105 (D), recognized markers of NE-MSCs, and negative for CD45 (E), known marker for hematopoietic stem cells. 10,000 events counted.

Figure 2: In vitro assessment of multipotency. BM-MSCs and NE-MSCs were induced to differentiate into osteocytes (alizarin red, A), chondrocytes (alcian blue, B) and adipocytes (red oil, C). Both BM-MSCs and NE-MSCs showed mineralization production at D14. NE-MSCs differentiated in chondrocyte at D14, while BM-MSCs at D21. BM-MSCs and NEMSCs also differentiated in adipocytes at D14.

Figure 3: (A) BCP and BG 3D constructs visualization by SEM at D2, D7, D14, and D21. BCP and BG had similar shape and size but different surface microstructures, BCP particles showed microporosity, whereas BG exhibited a smooth surface. Both types of MSCs adhered more rapidly to $B G$ than to $B C P$. BM-MSCs synthesized an extracellular matrix after D14, which seem to be more abundant on $\mathrm{BG}$ than on $\mathrm{BCP}$, but mineralization was only observed on $\mathrm{BCP}$. NEMSCs produced also an abundant, but fragile, mineralized extracellular matrix on both biomaterials (less on BG than on BCP). (B) Comparative adhesion and viability of the two MSC types on biomaterials. Using fluorescent stains for living (green) and dead (red) cells, BMMSCs and NE-MSCs viability was kinematically assessed. Dead cells (white arrows) are observed only on BCP biomaterial. Death of newborn stem cells was only observed at D21 while apoptosis/necrosis of adult stem cells starts as soon as D2. 
Figure 4: Influence of the biomaterial on cell proliferation. BM-MSCs and NE-MSCs were seeded on 2D plastic at two different densities: 500 (A) and 5,000 cell/ $\mathrm{cm}^{2}$ (B). (C-E) Both cell types were seeded on BCP and BP and cell metabolic activity monitored over a period of three weeks using alamarBlue ${ }^{\circledR}$ assay. On plastic, NE-MSC density increases more rapidly during the first week but an equalization occurs at the end of the second week. During the third week, cell death induces a reduced confluence. C-E graphs indicate that, when compared to BCP, BG biomaterial favors BM-MSC proliferation. Controls corresponds to cell-free biomaterials (background noise of alamar blue).

Figure 5: (A) Comparative production of collagen. In osteogenic conditions, BM-MSCs increasingly produce collagen while the opposite is observed for NE-MSCs. Comparative production of ALP by both cell types, at D7. BM-MSCs and NE-MSCs were cultivated either on plastic (B) or biomaterials (C). BM-MSCs express ALP only when cultivated on BG biomaterial while NE-MSCs produce ALP on both biomaterials. For NE-MSCs, ALP production is higher in proliferative medium.

Figure 6: Quantification of alkaline phosphatase (ALP). For the purpose of comparison, the total amount of DNA, at D1 and D7, was quantified for both cell types BM-MSCs and NE-MSCs, in proliferative and osteogenic conditions (A). The total amount of $A L P$ in each condition was assessed (B) and then compared to the amount of DNA (C). NE-MSCs express an important amount of $A L P$ when grown on $\mathrm{BG}$, in osteogenic conditions. For BM-MSCs, $A L P$ expression is marginal except at D1 on BCP biomaterial. 
Figure 7: Comparative expression of ossification-related genes. Using RTqPCR, the expression of genes coding for RUNX2, BSP, COL1A1, OC, BMP2, BMP4, ALP, and OP was measured for each cell type on plastic, BG and BCP biomaterials. On BG biomaterial, NE-MSCs overexpress the genes coding for BSP, OC and OP (A-C) and BM-MSCs the gene coding for RUNX2 (D).

Figure 8: Expression of other ossification-related genes $\operatorname{COL1A1}(\mathbf{A}), A L P(\mathbf{B}), B M P 2(\mathbf{C})$, and BMP4 (D) was measured by RTqPCR for each cell type on plastic, BG and BCP biomaterials. No data to D7 on BCP because the cells did not proliferate, and we obtained too little RNA.

Figure 9: In vivo bone formation, 8 weeks after subcutaneous implantation in nude mice. (A) BM-MSCs give rise to osteocytes, stained with Masson trichrome (red arrows), but not NEMSCs and cell-free BCP biomaterial. (B) The same finding is observed when the implants are analyzed with an electronic microscope (red arrows). (C) No bone formation is observed when cells were loaded in BG biomaterial. (D) Table summarizing the findings. 


\section{A}

Control BCP without cell

\section{$\underline{1 \mathrm{~mm}}$}

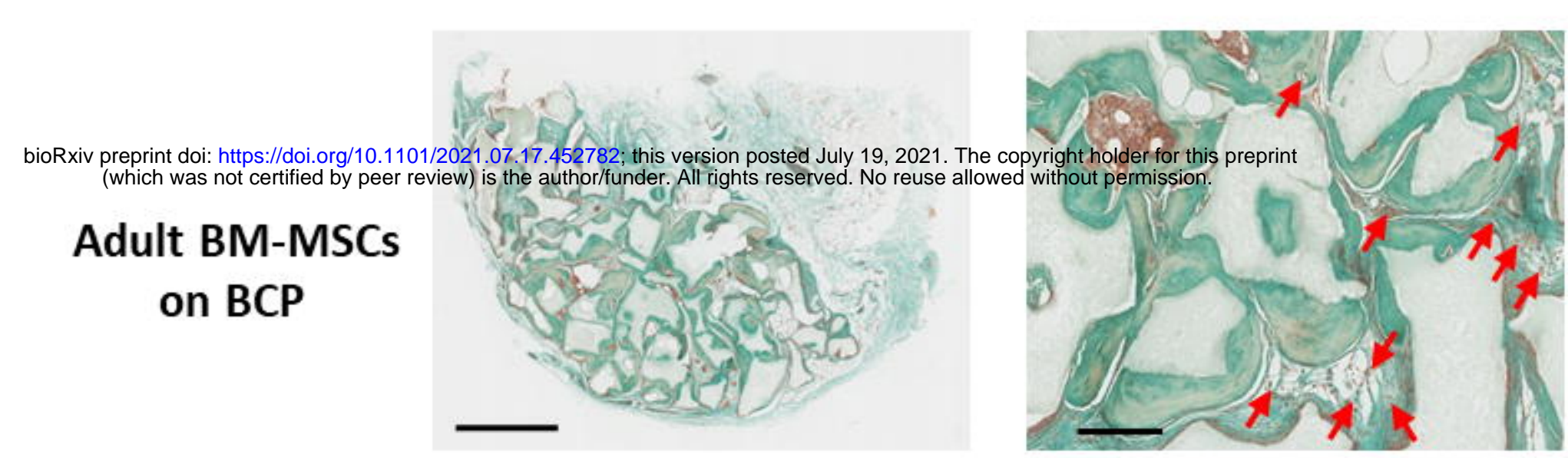

Newborn NE-MSCs on BCP

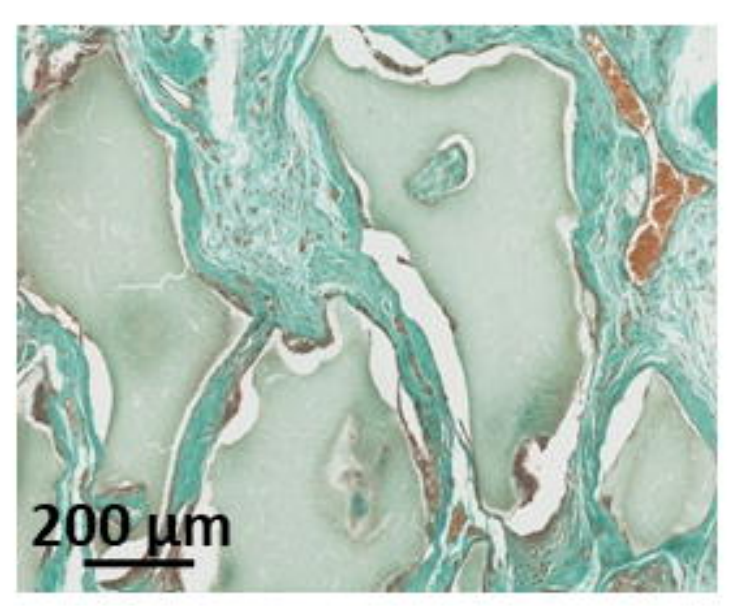

B
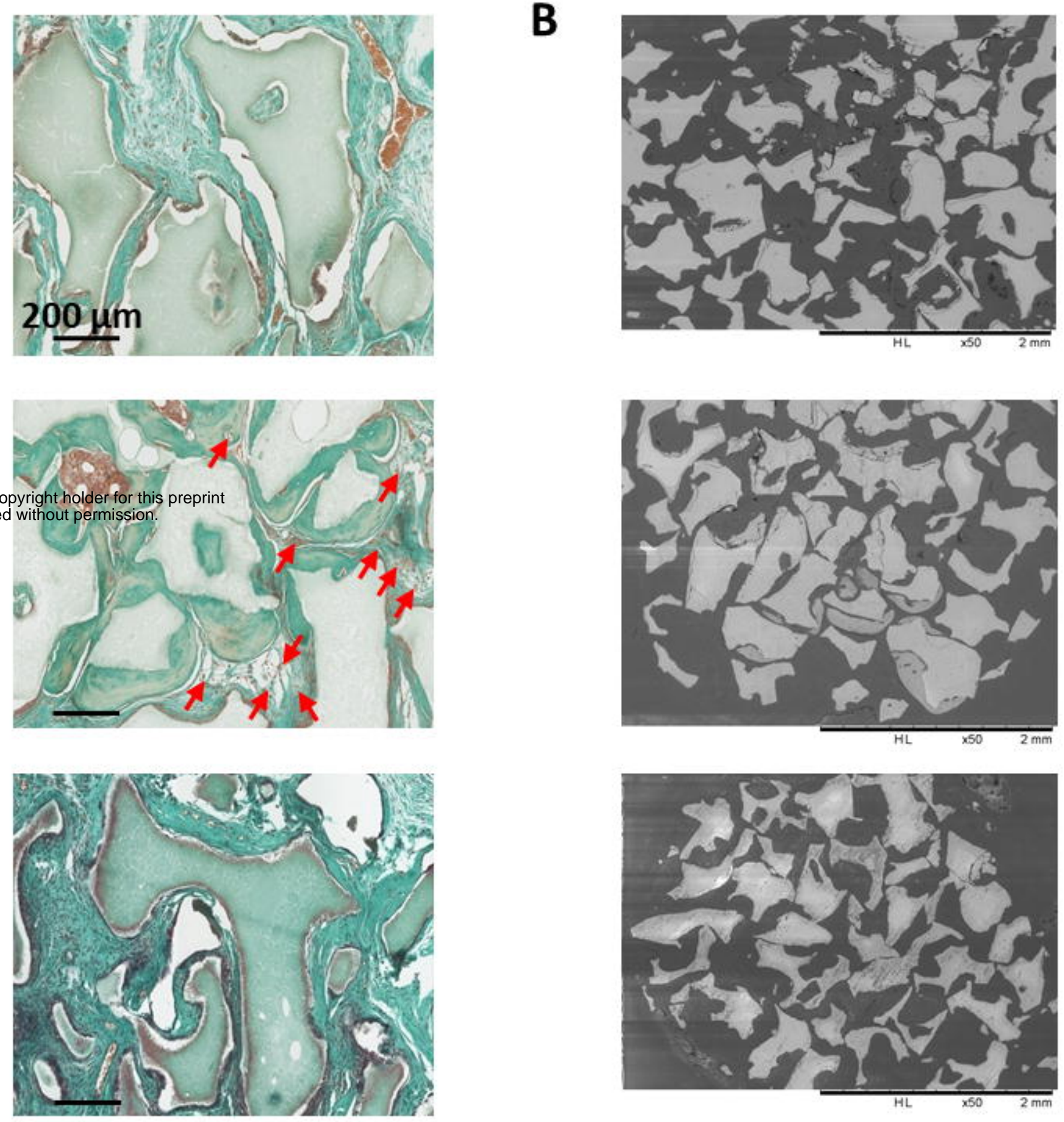

Control BG without cell

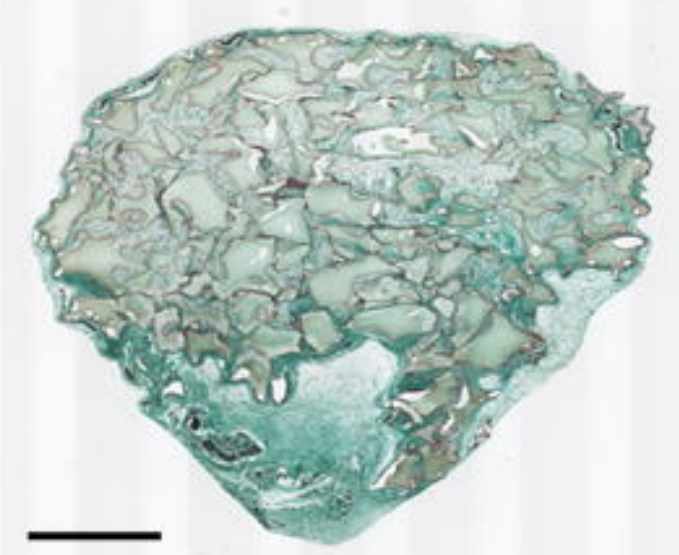

C
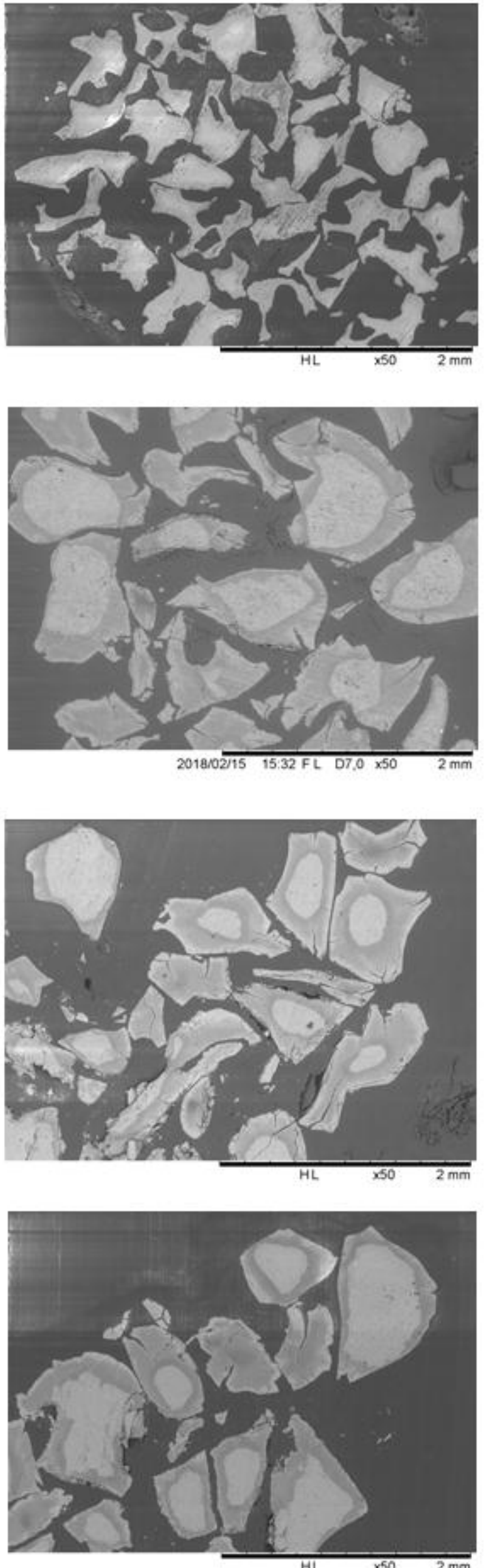

Adult BM-MSCs on BG

Newborn NE-MSCs on BG
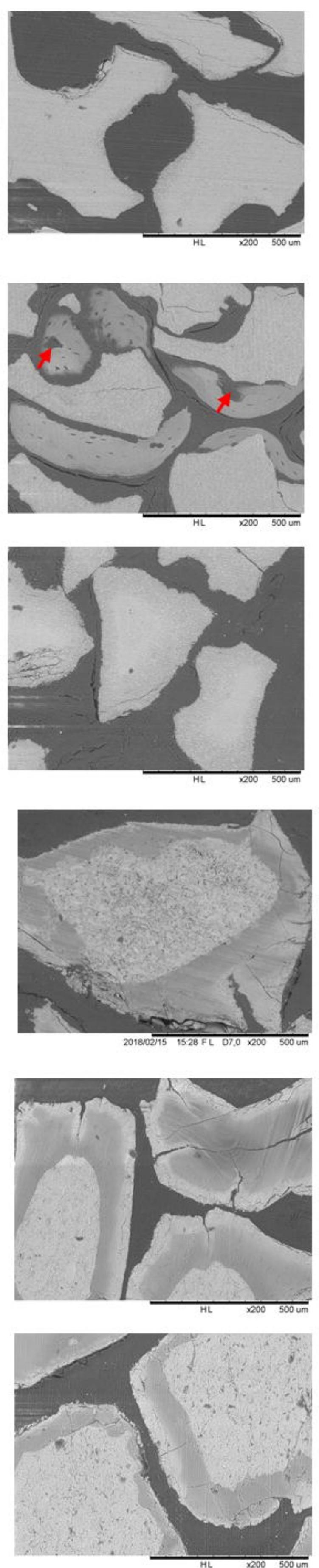
Controls
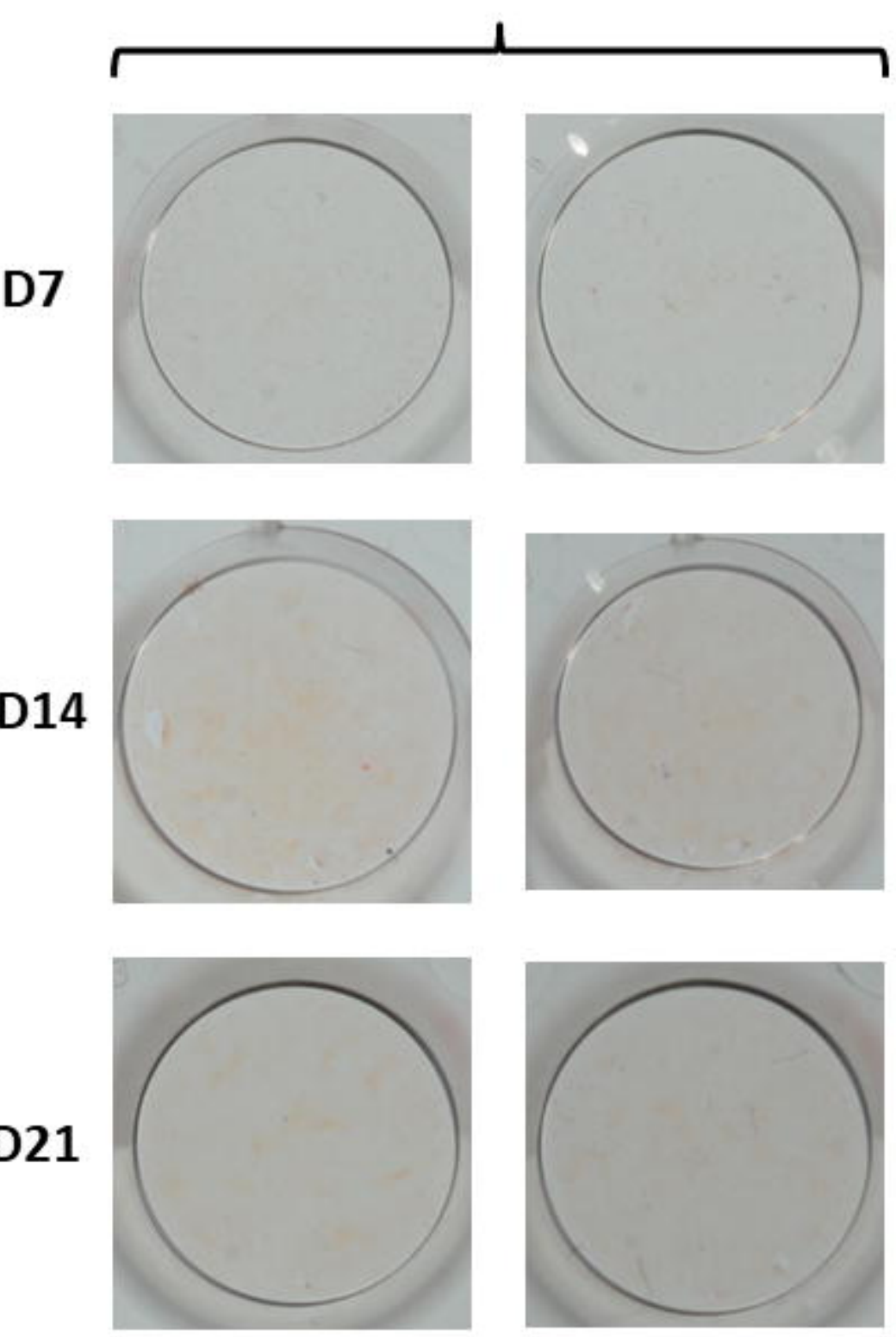

Adult BM-MSCs
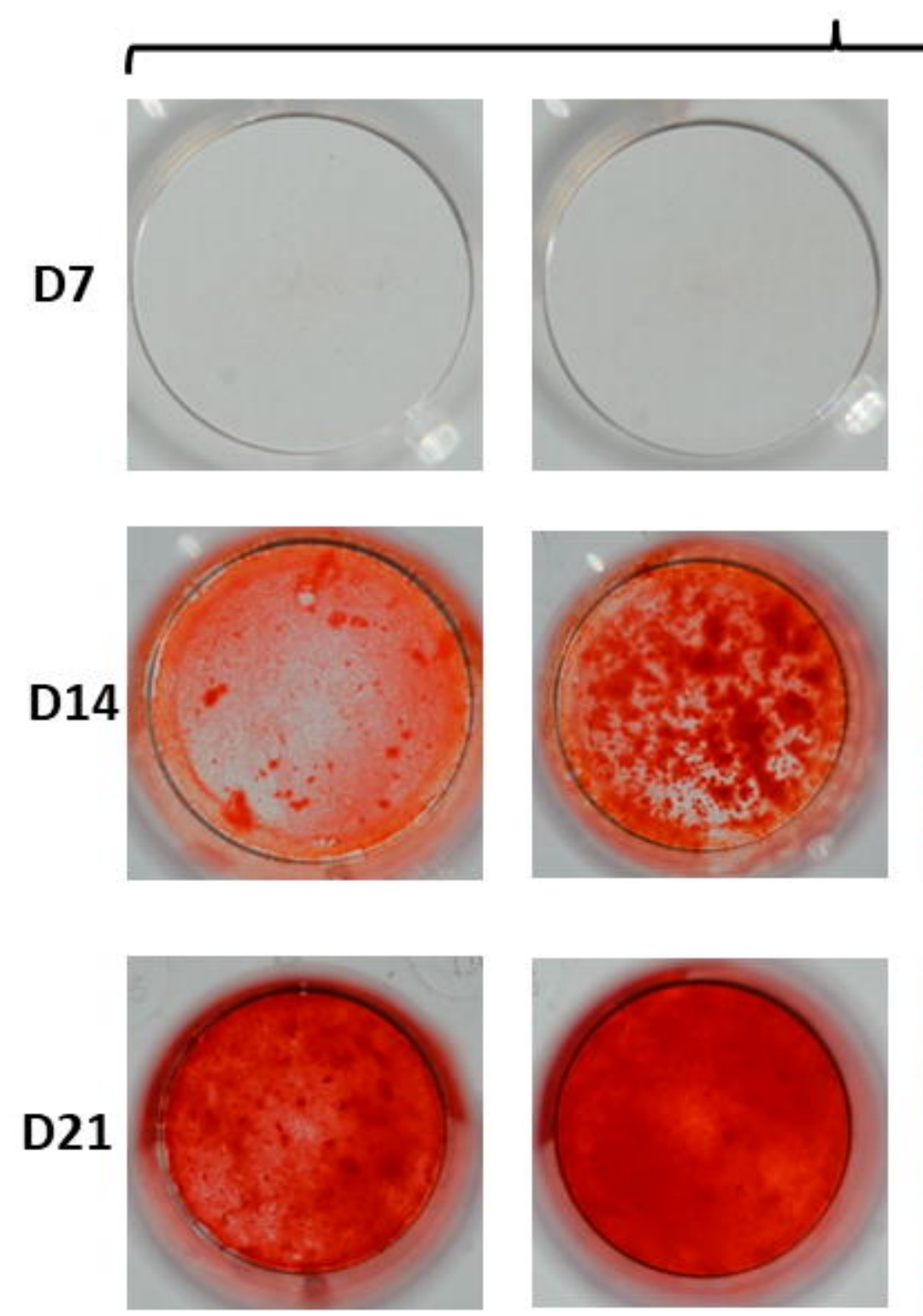
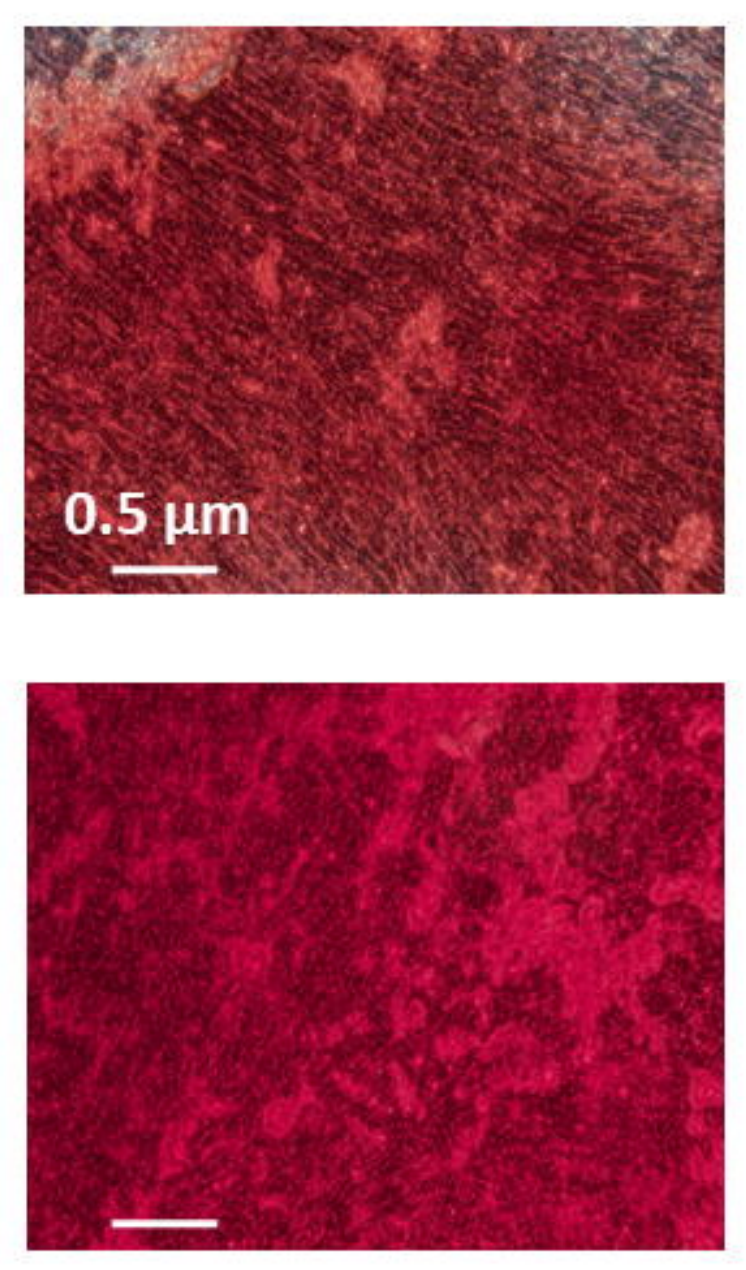

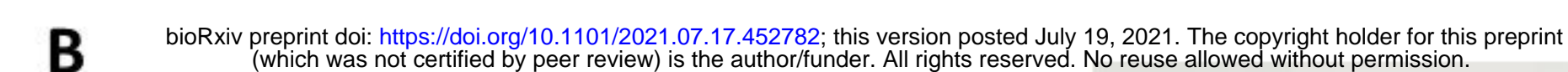

D14
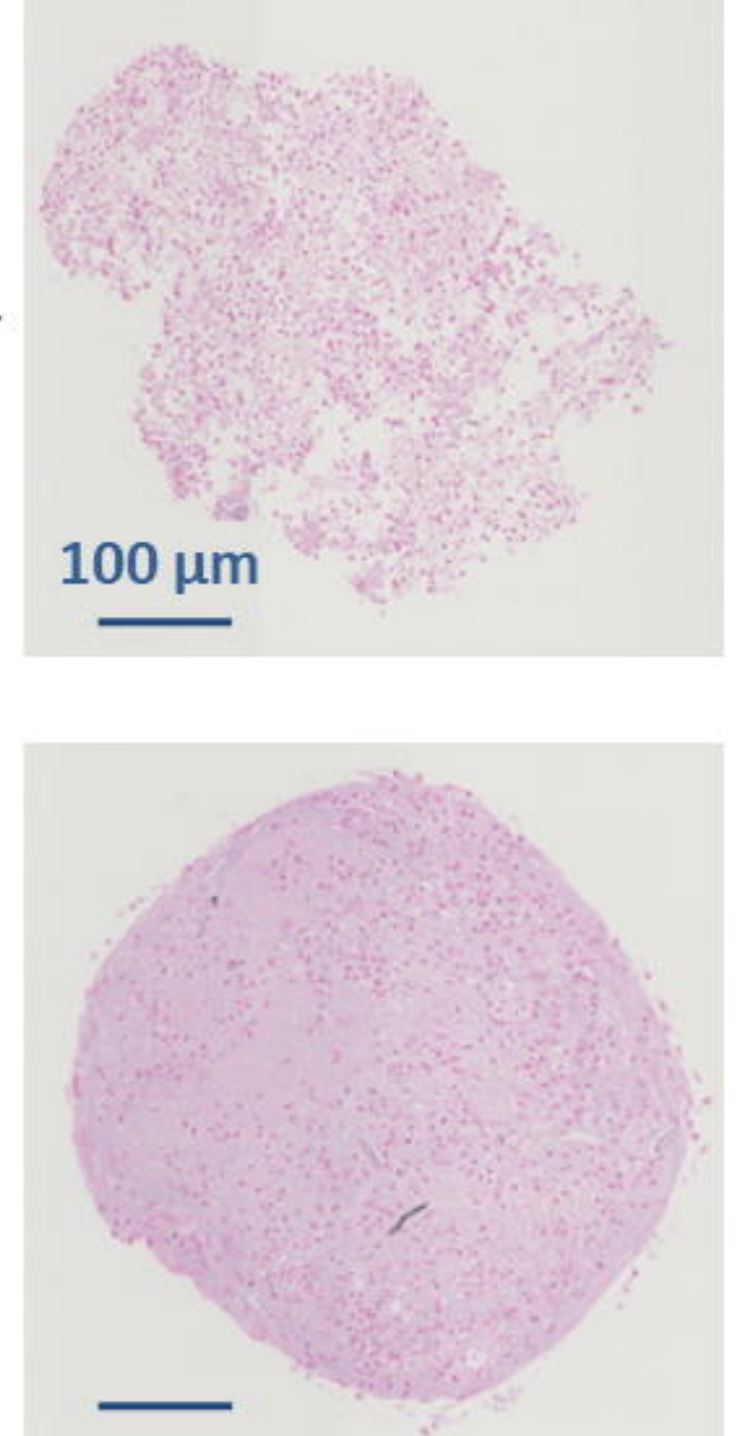

C
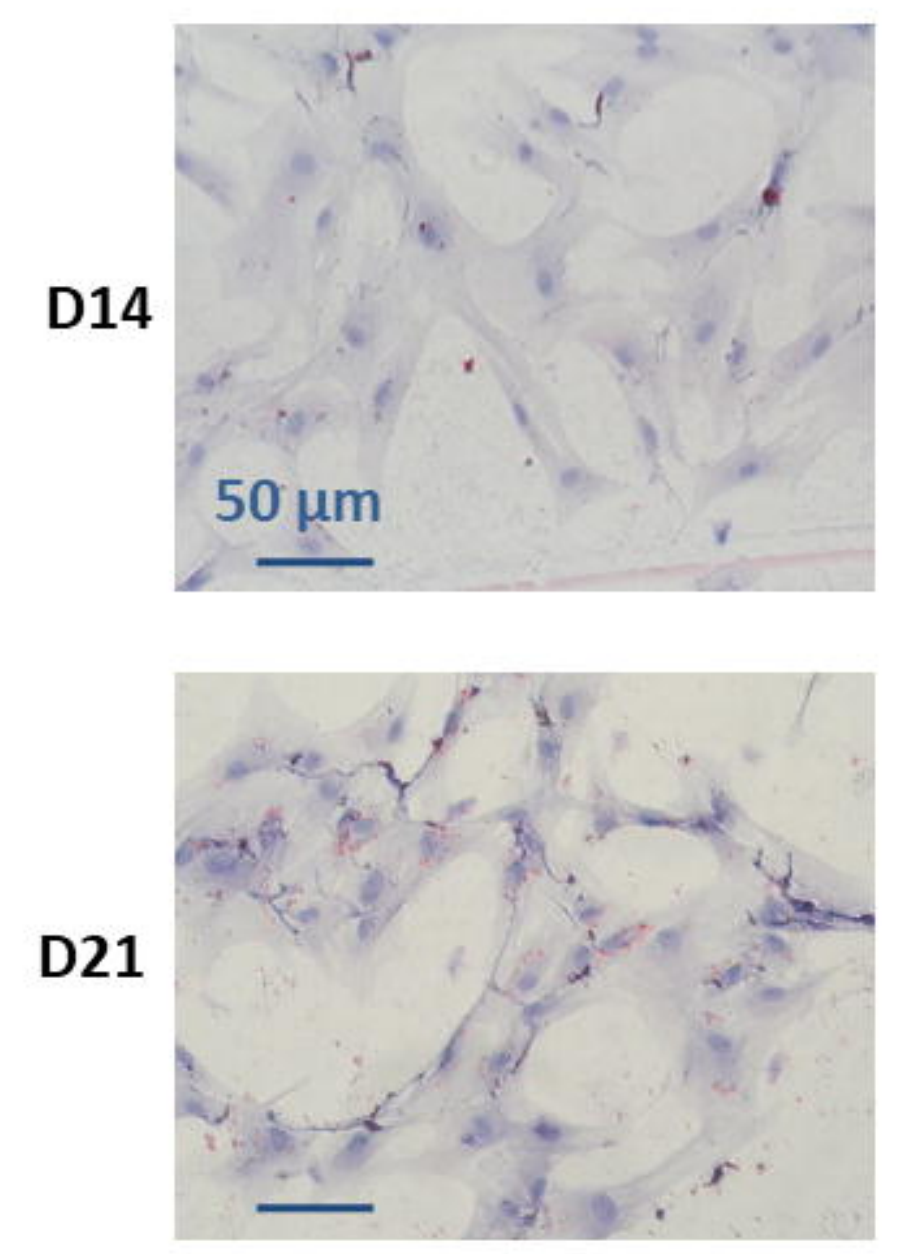
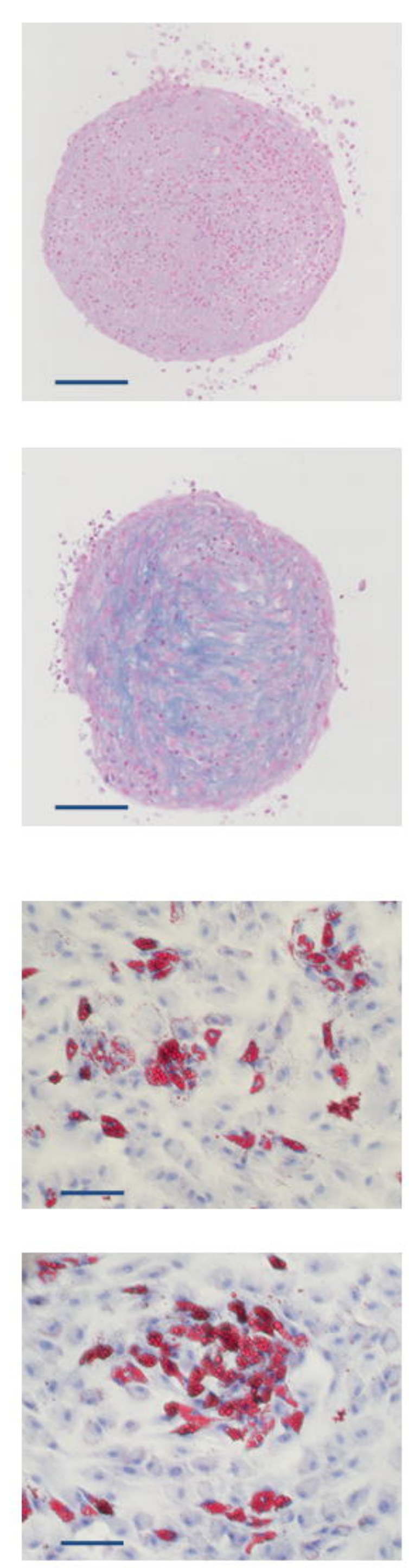

Newborn NE-MSCs
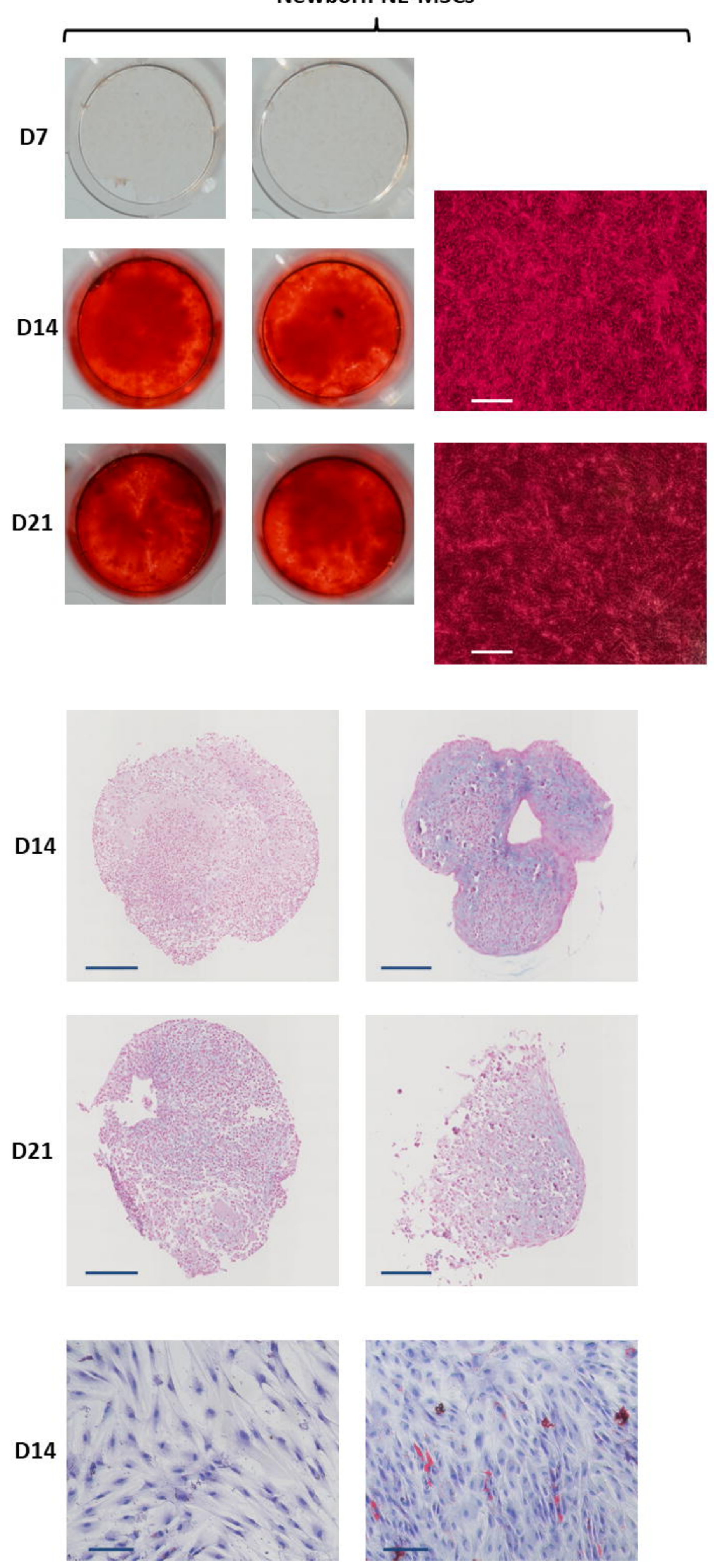

D21
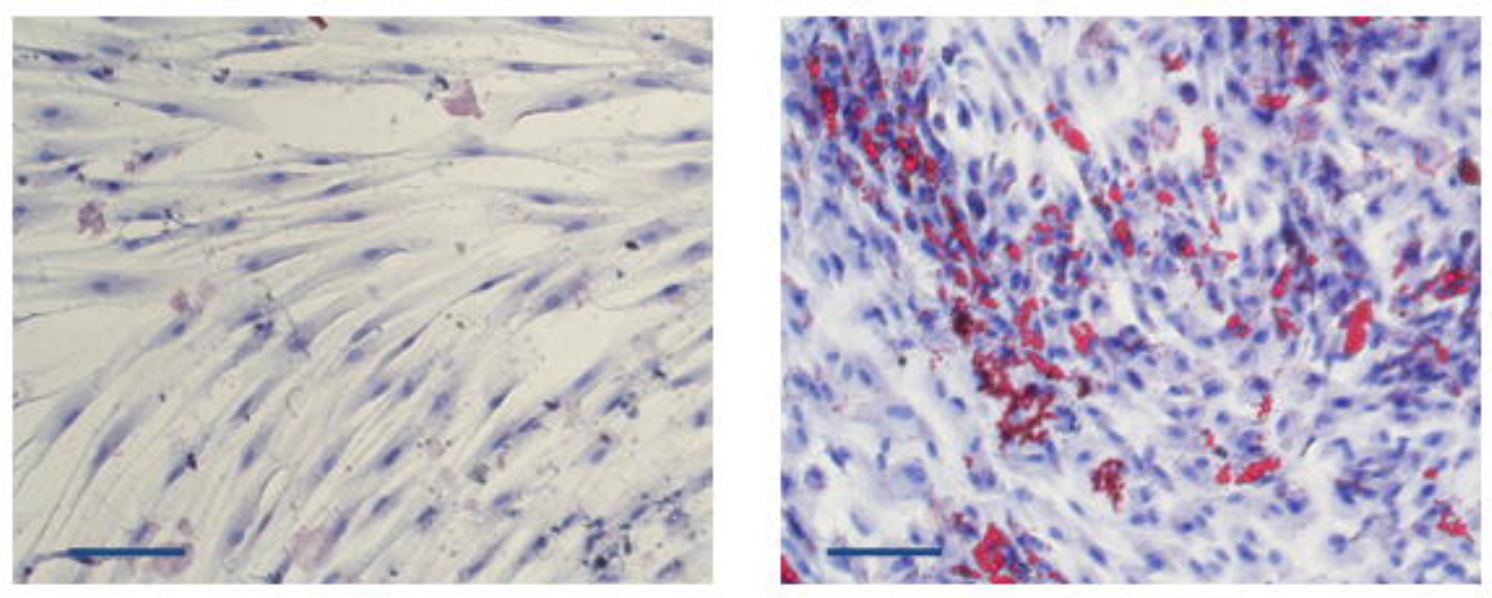

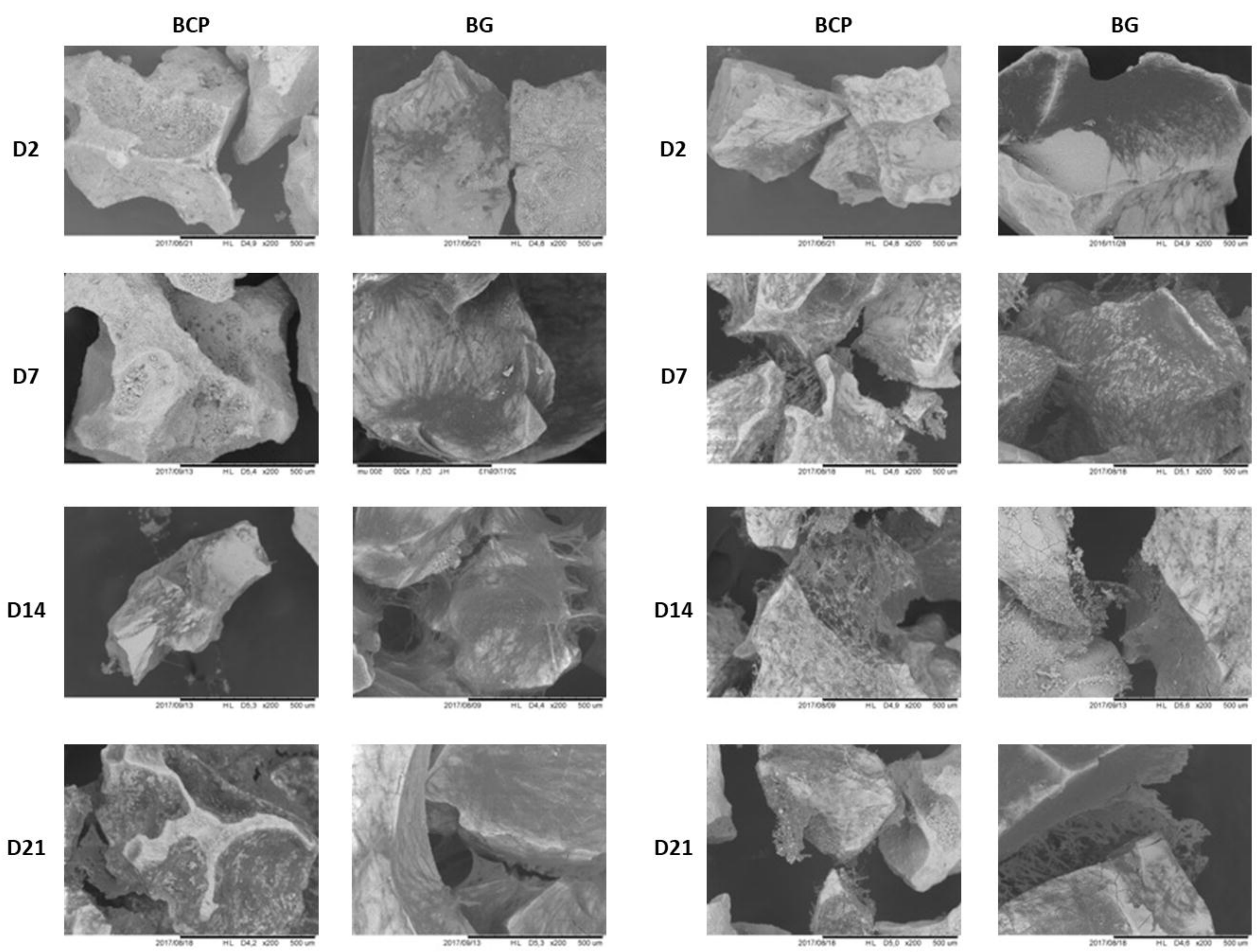

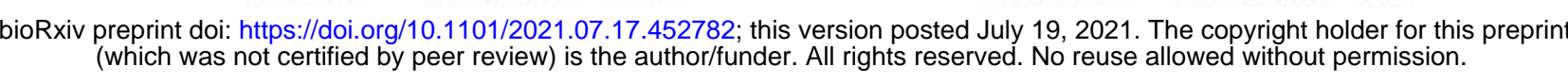

B

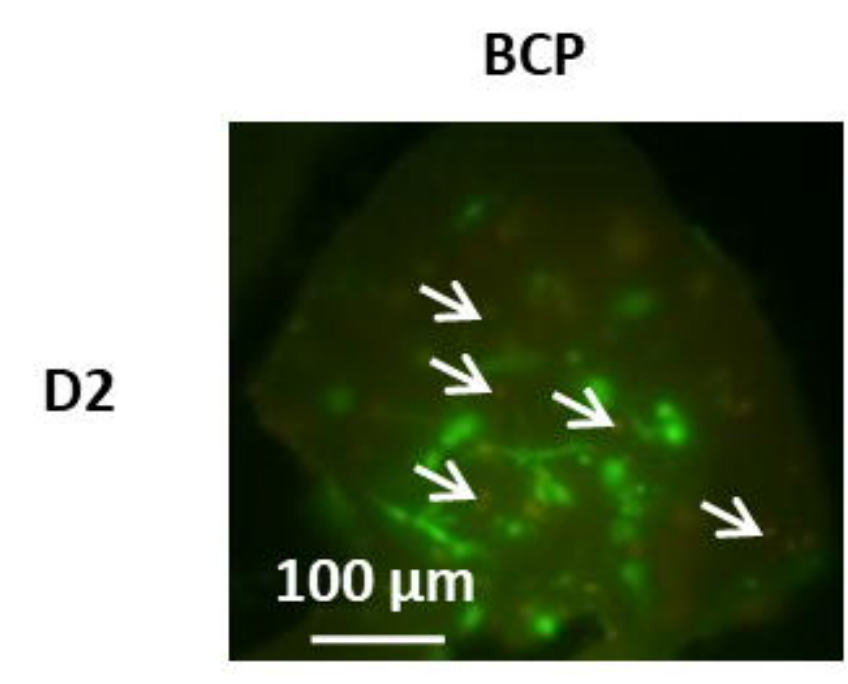

D7

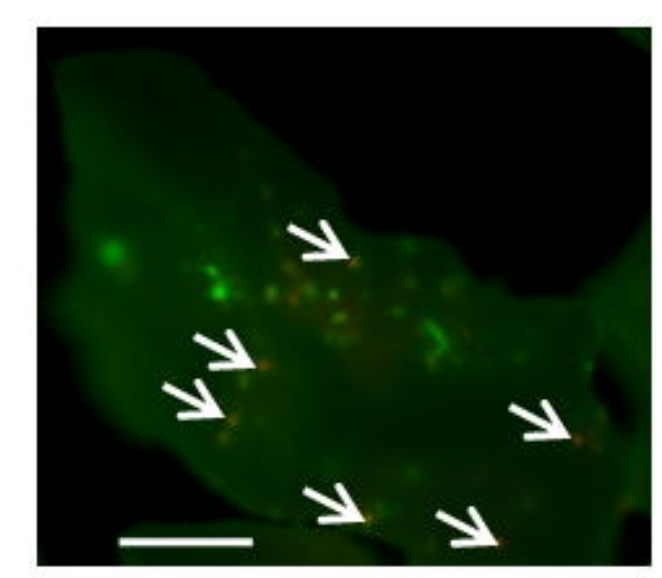

D14
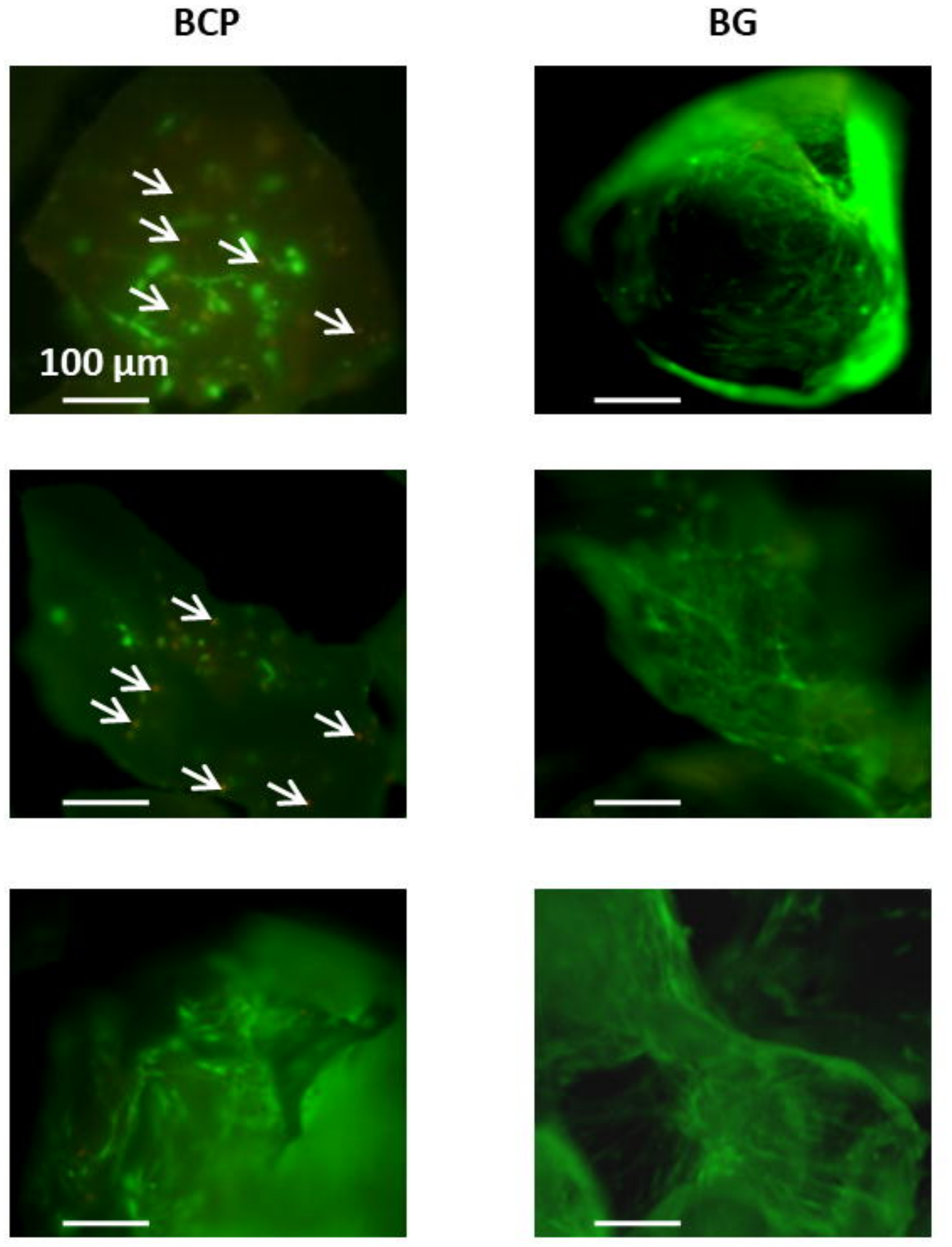

D2

D7

D14
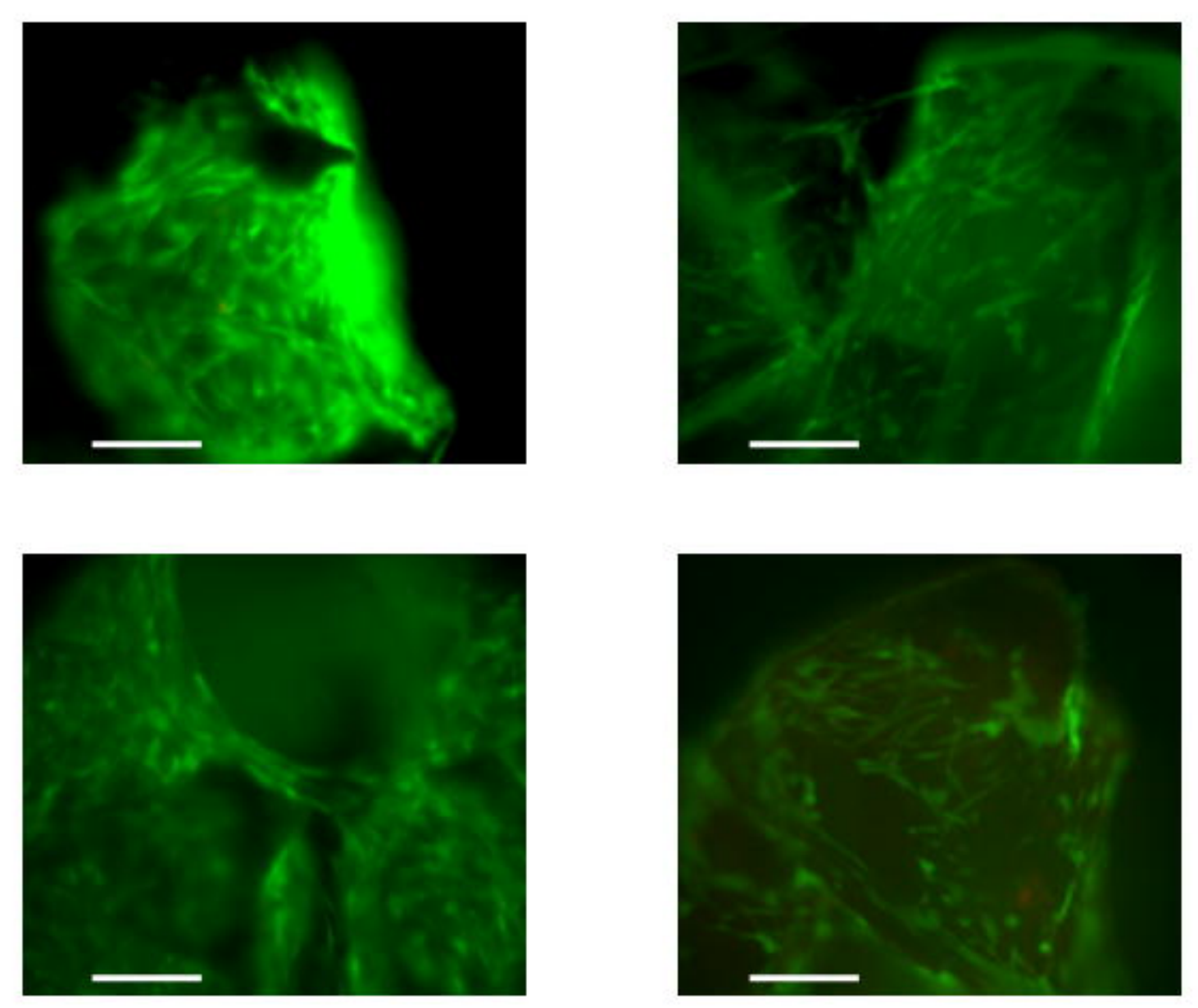

D21
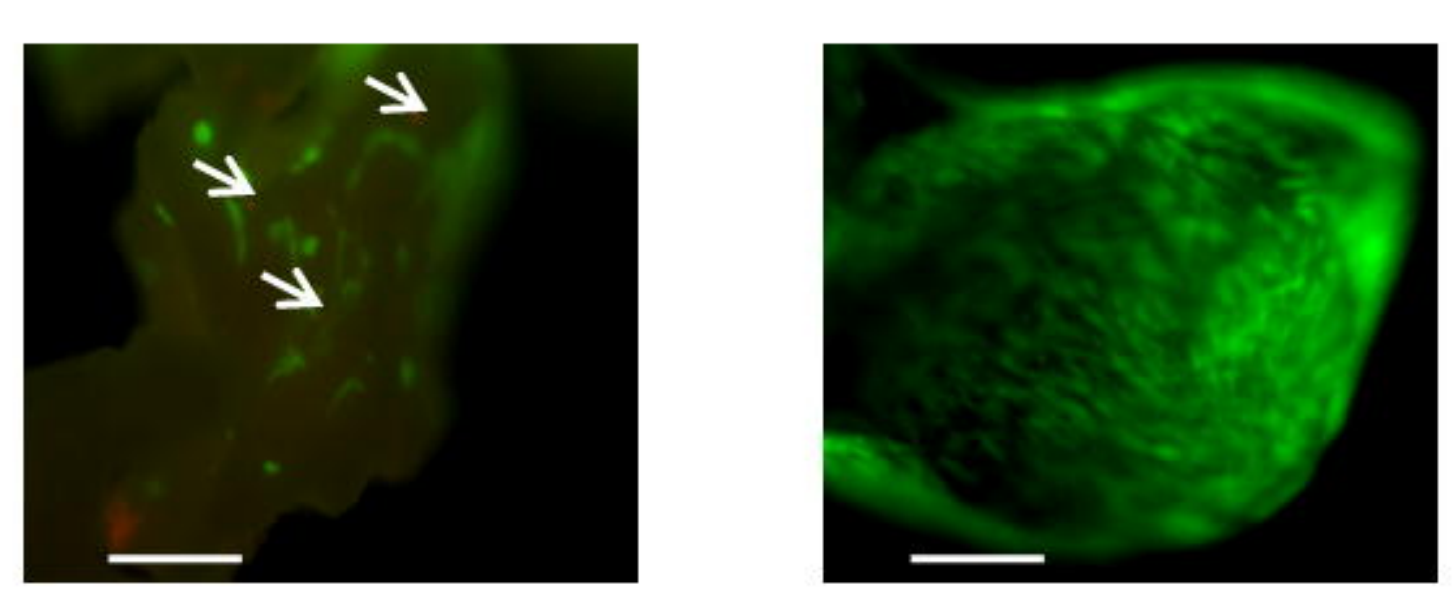


\section{A}

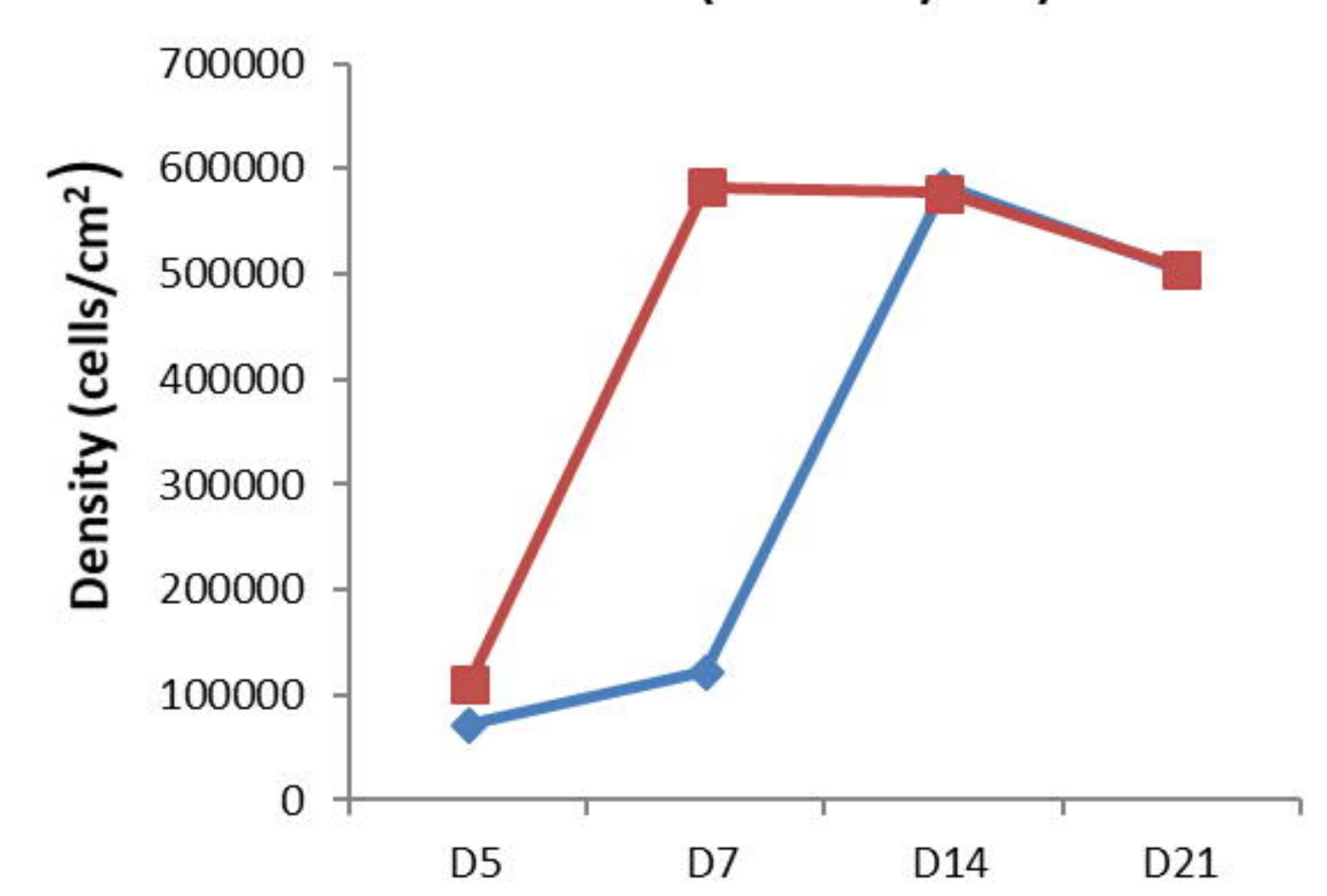

B

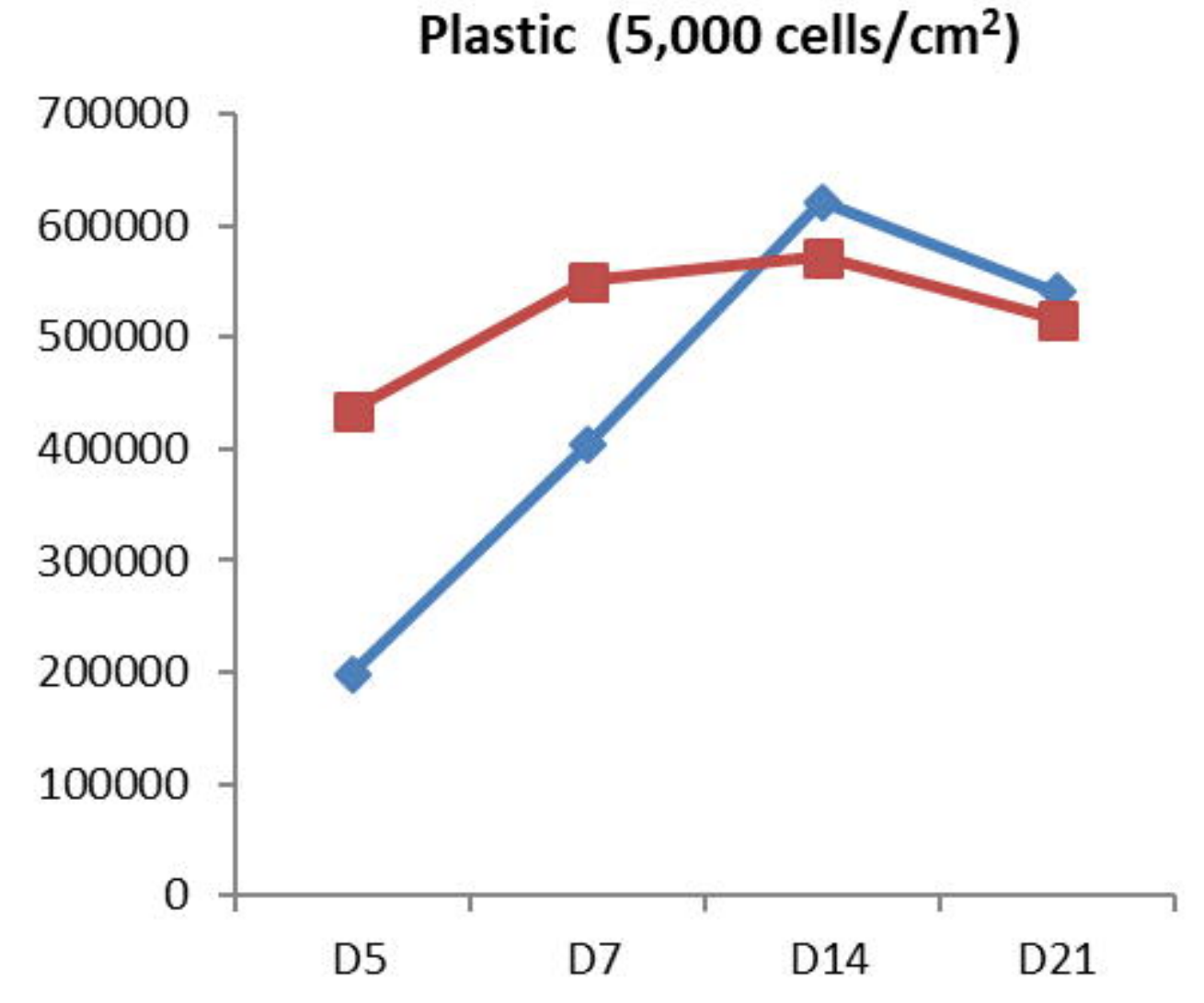

+ mantmanse

$=$

BCP

BG

\section{C}

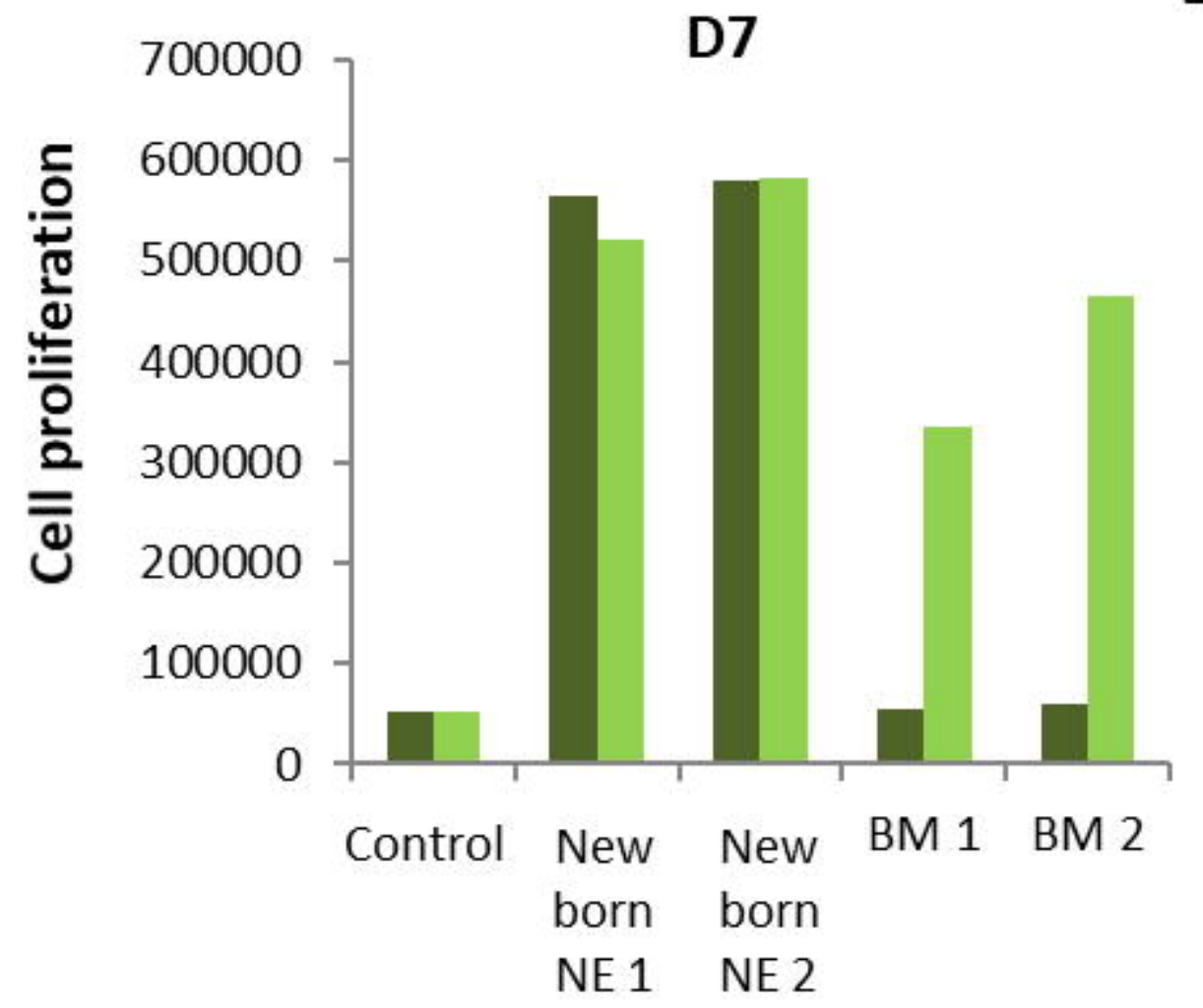

D

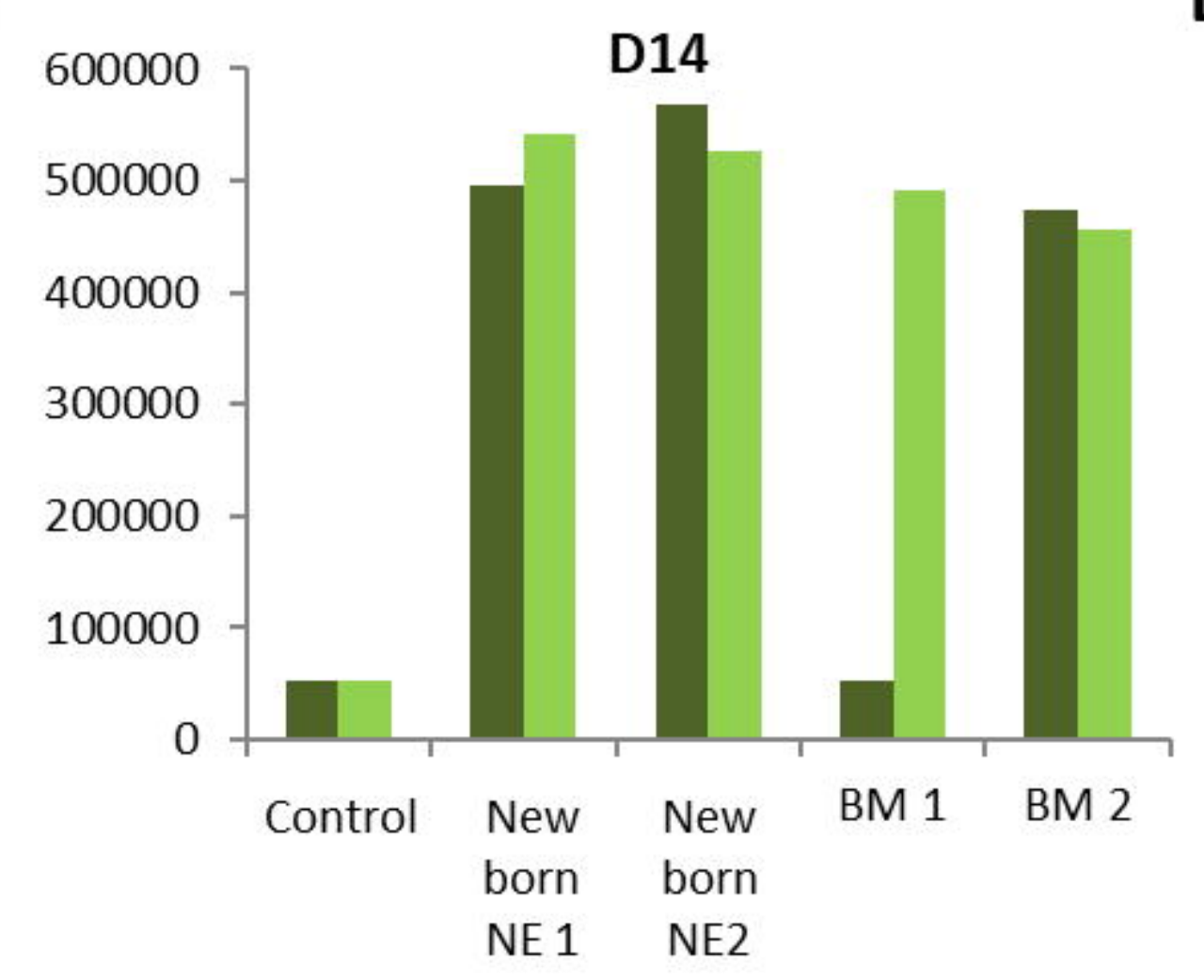

E

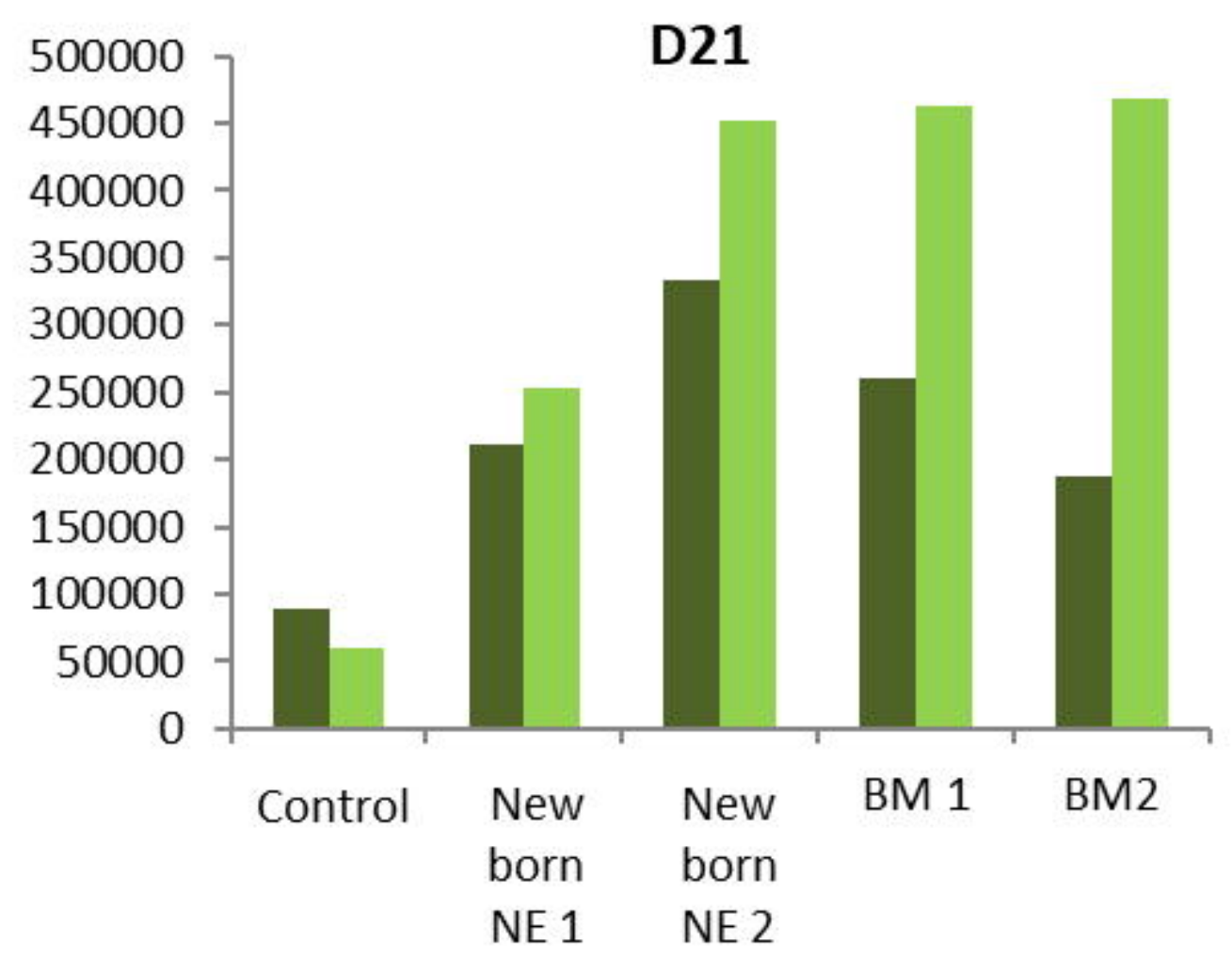


Proliferative medium

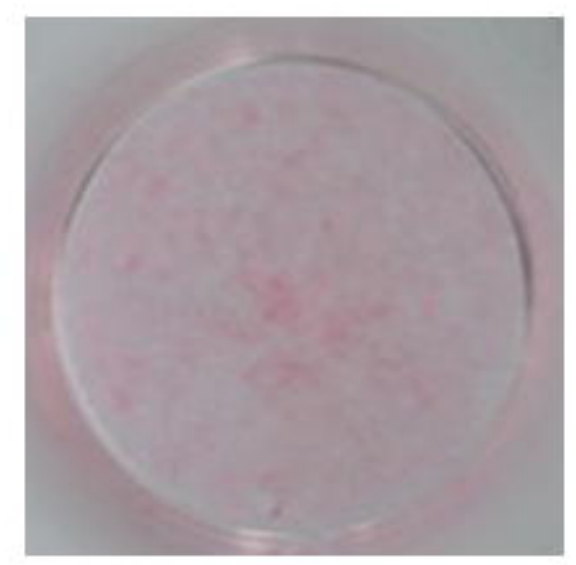

D14

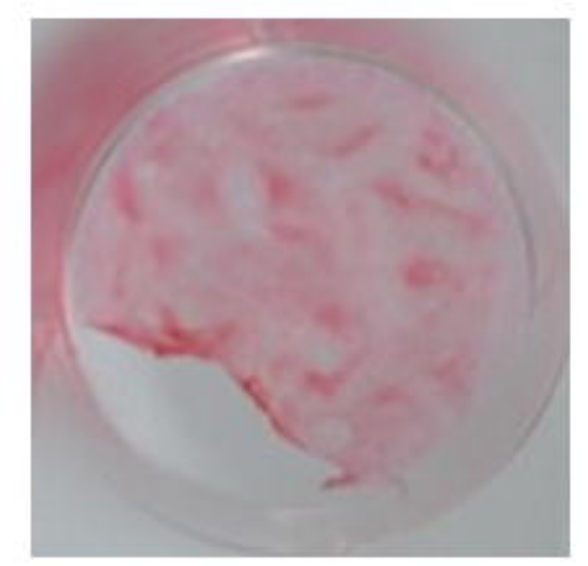

D21
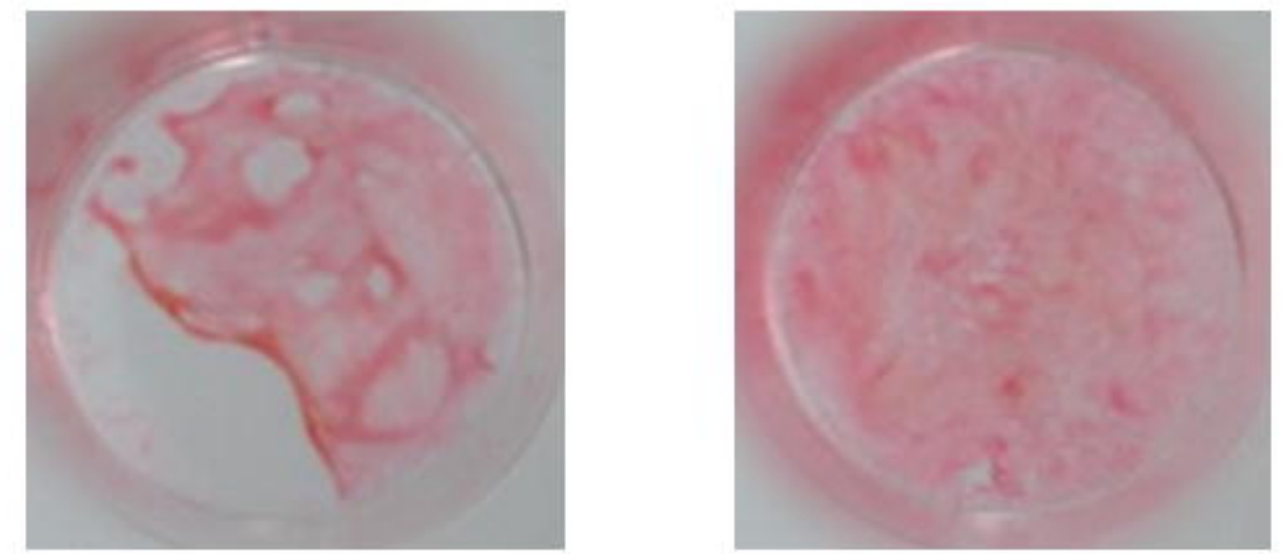

Adult BM-MSCs

B

Proliferative medium

Osteogenic medium

\section{D7}
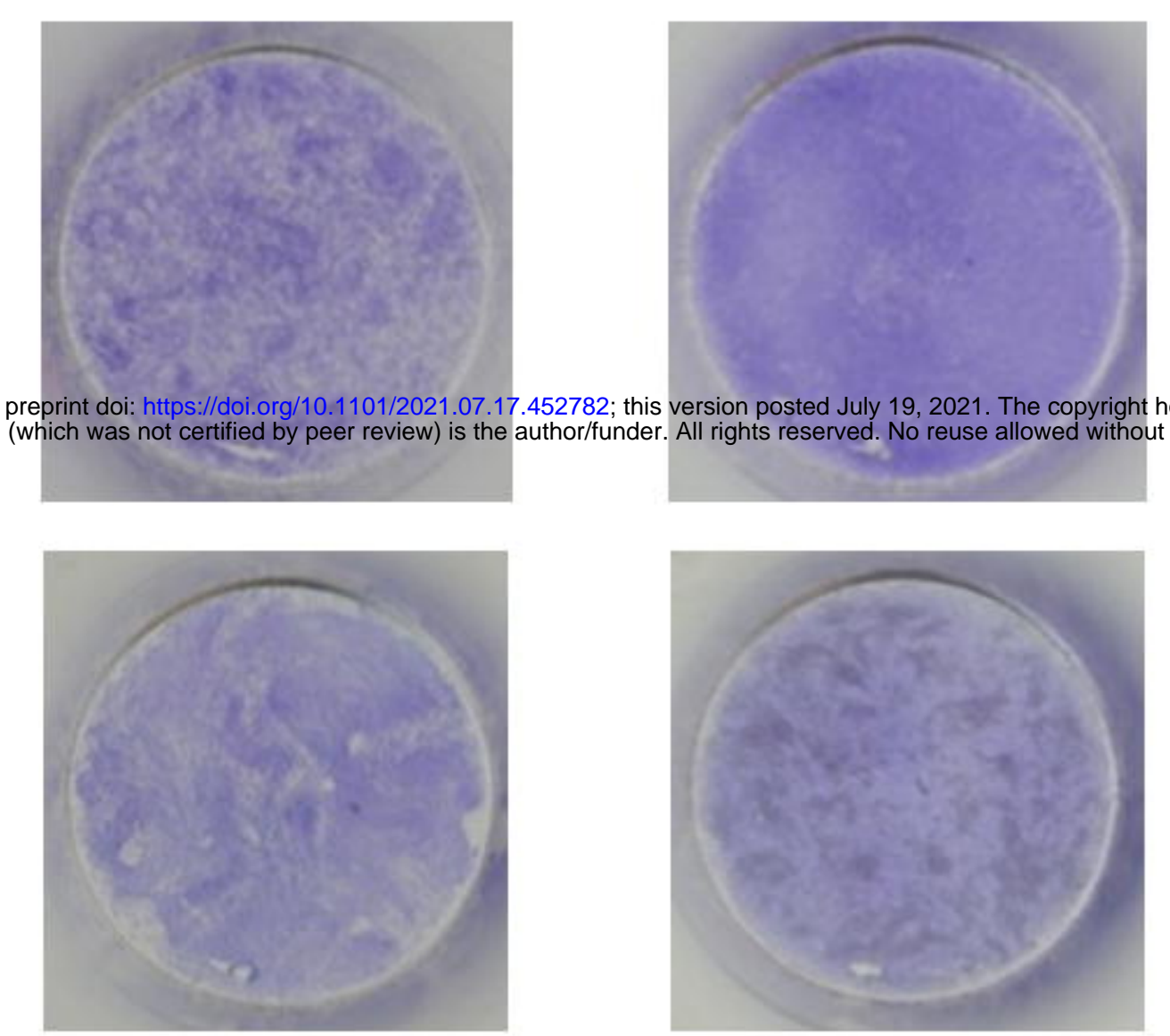

C

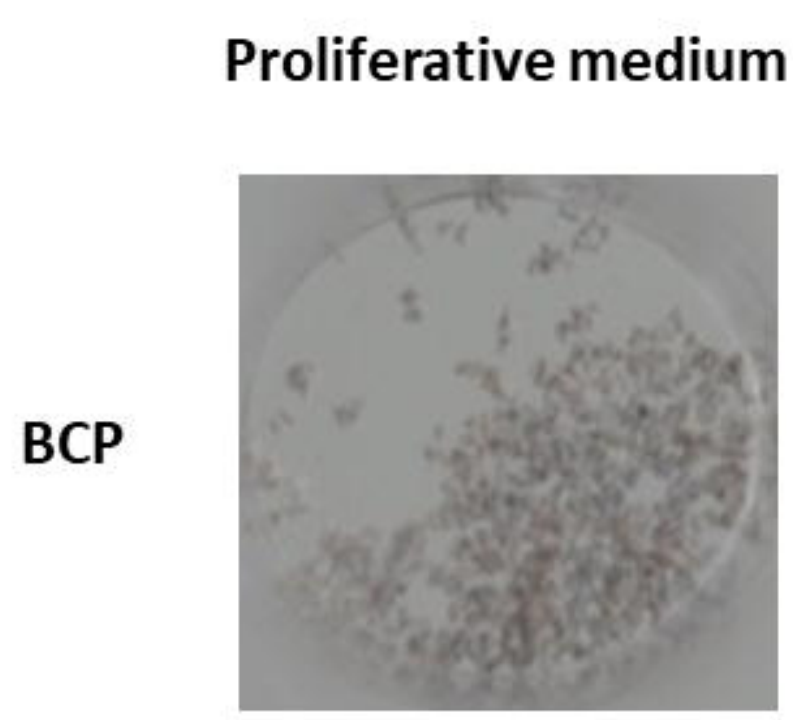

Adult BM-MSCs

Proliferative medium

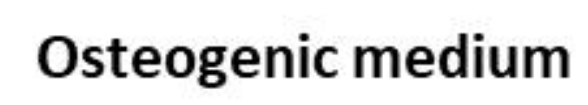

BG
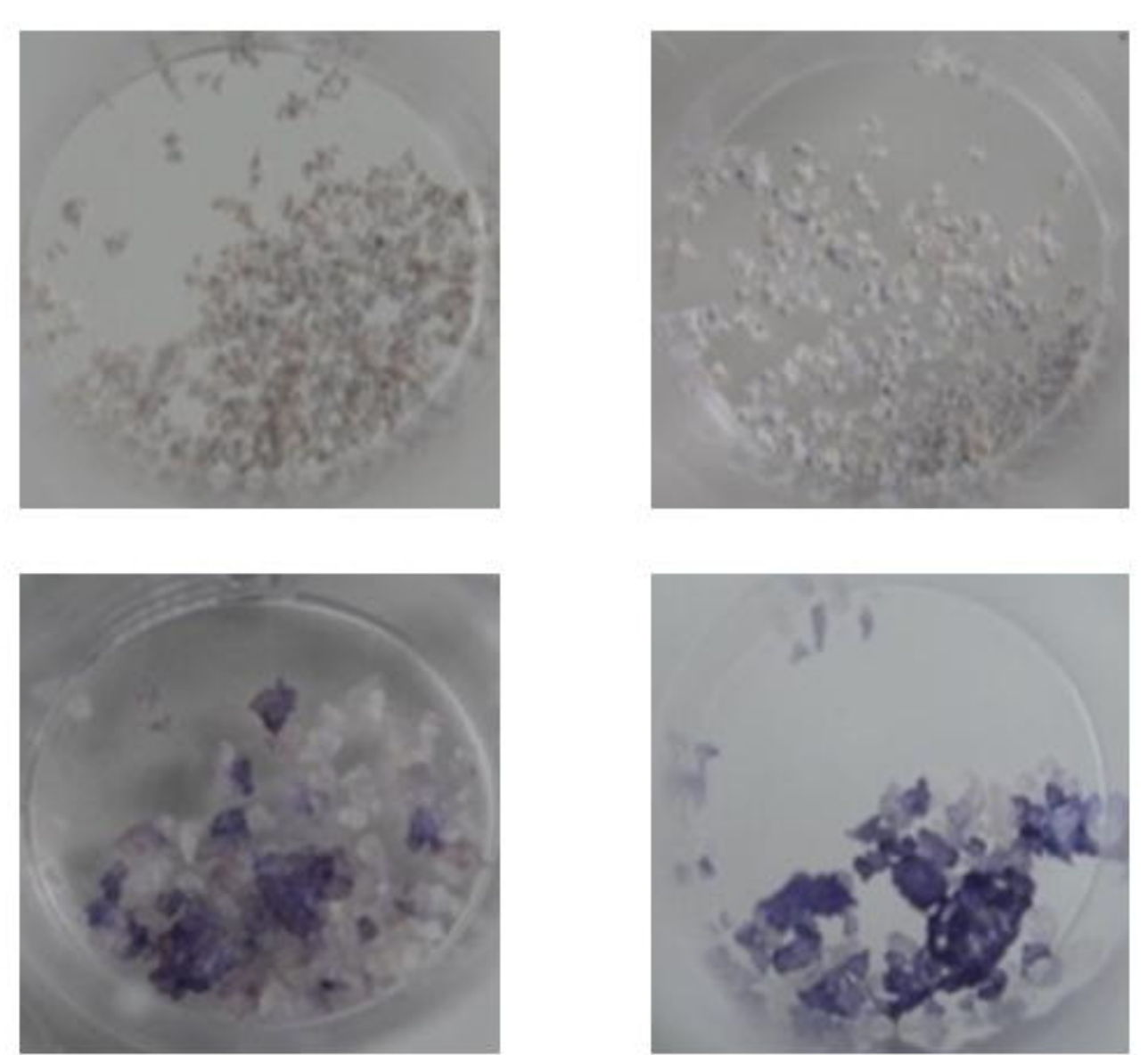

D14

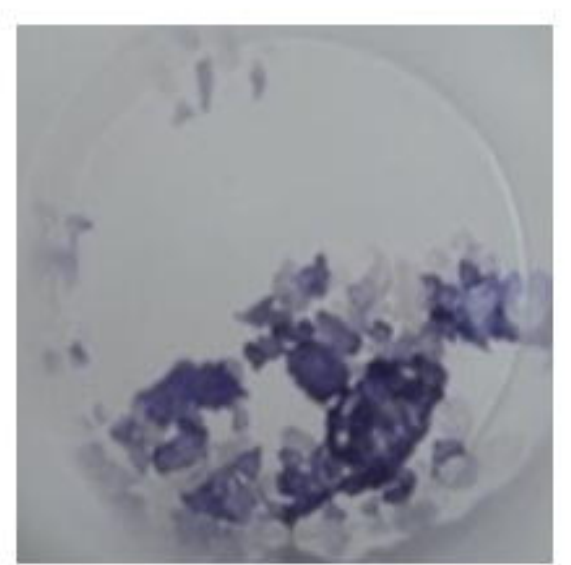

BG

Proliferative medium

Osteogenic medium

D7

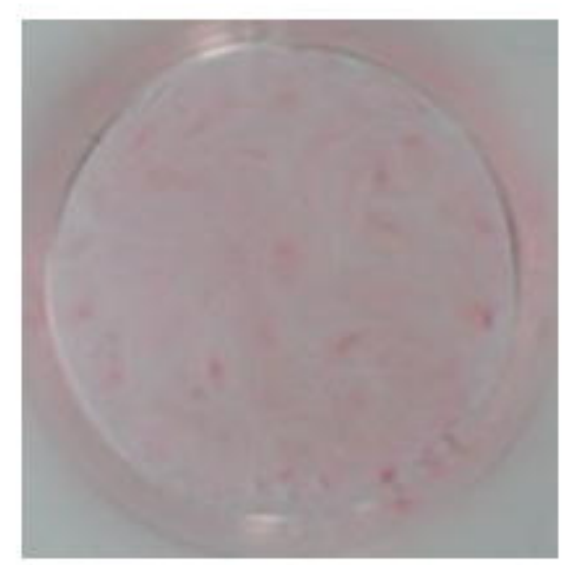

D14

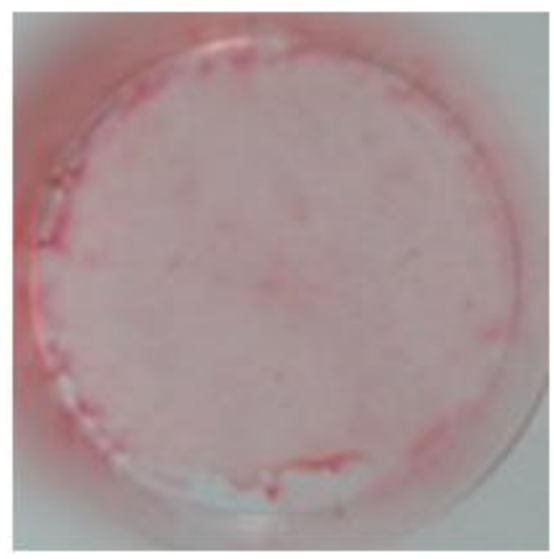

D21

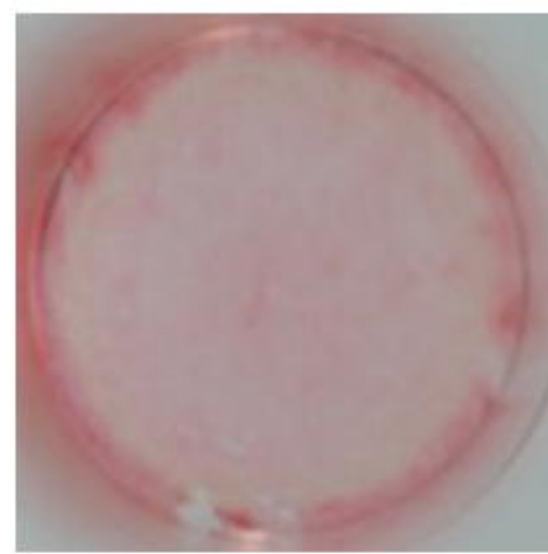

Newborn NE-MSCs

Proliferation medium

Osteogenic medium

D7
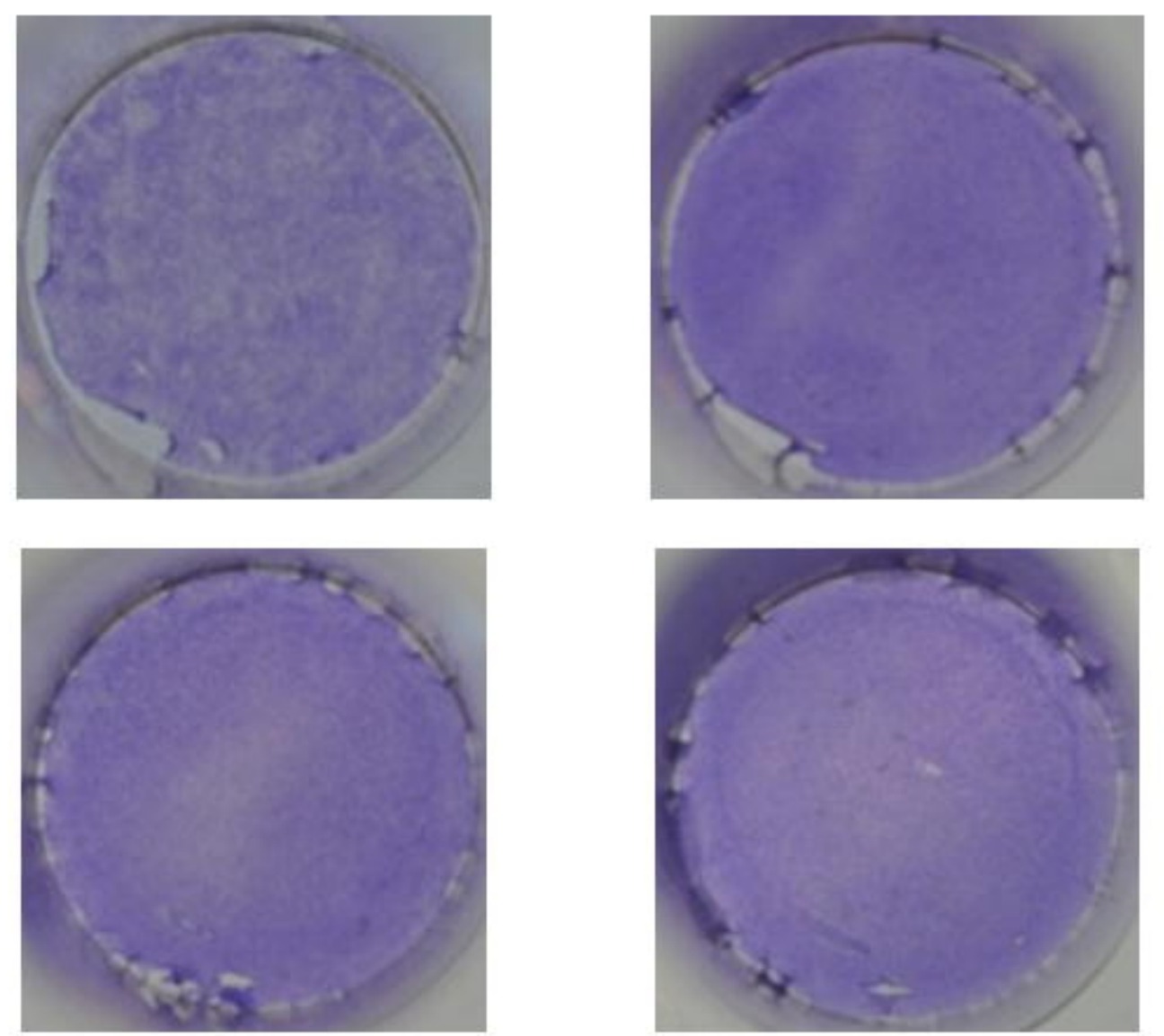

Newborn NE-MSCs

Proliferation medium

Osteogenic medium

BCP
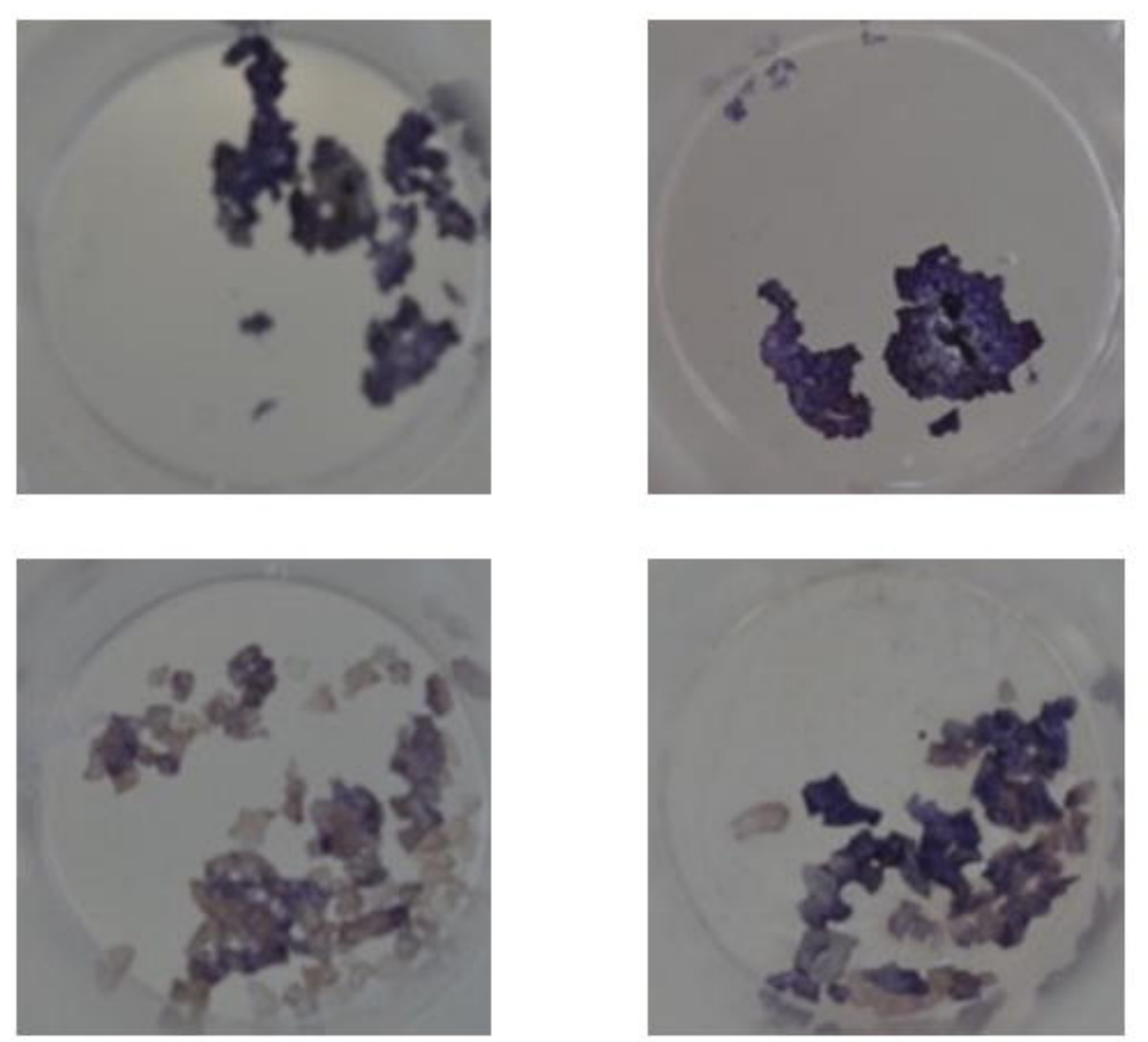


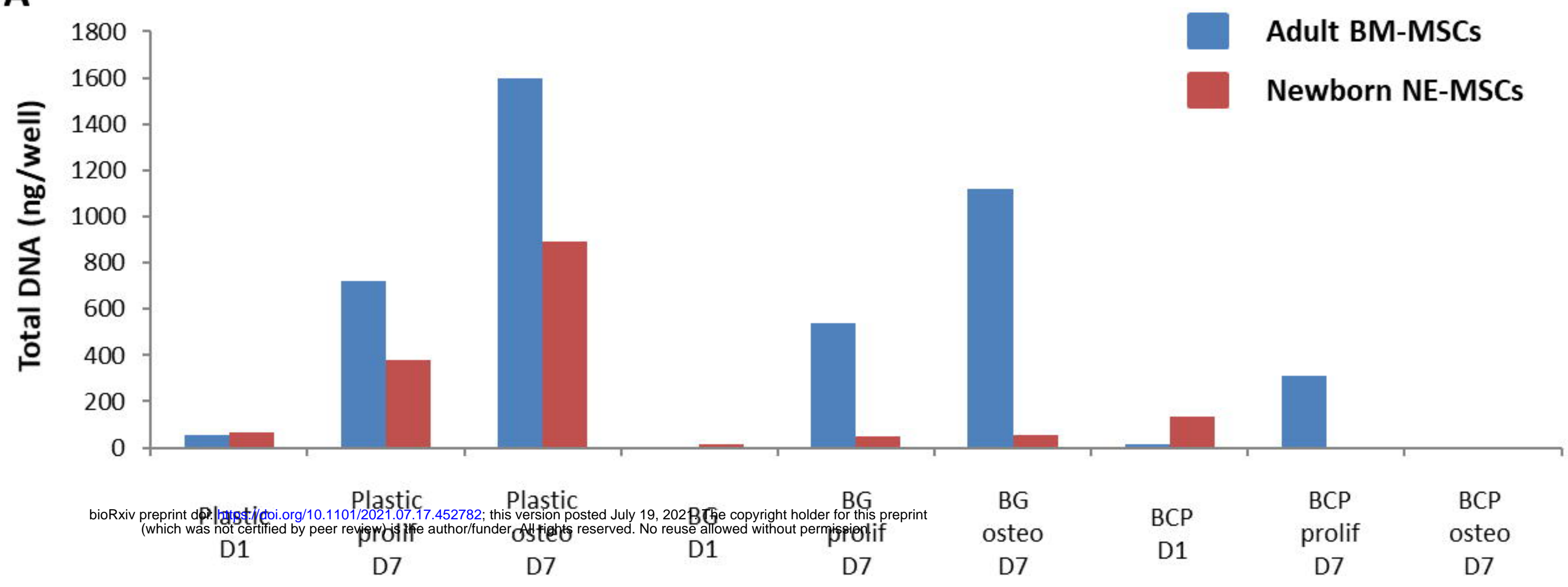

B

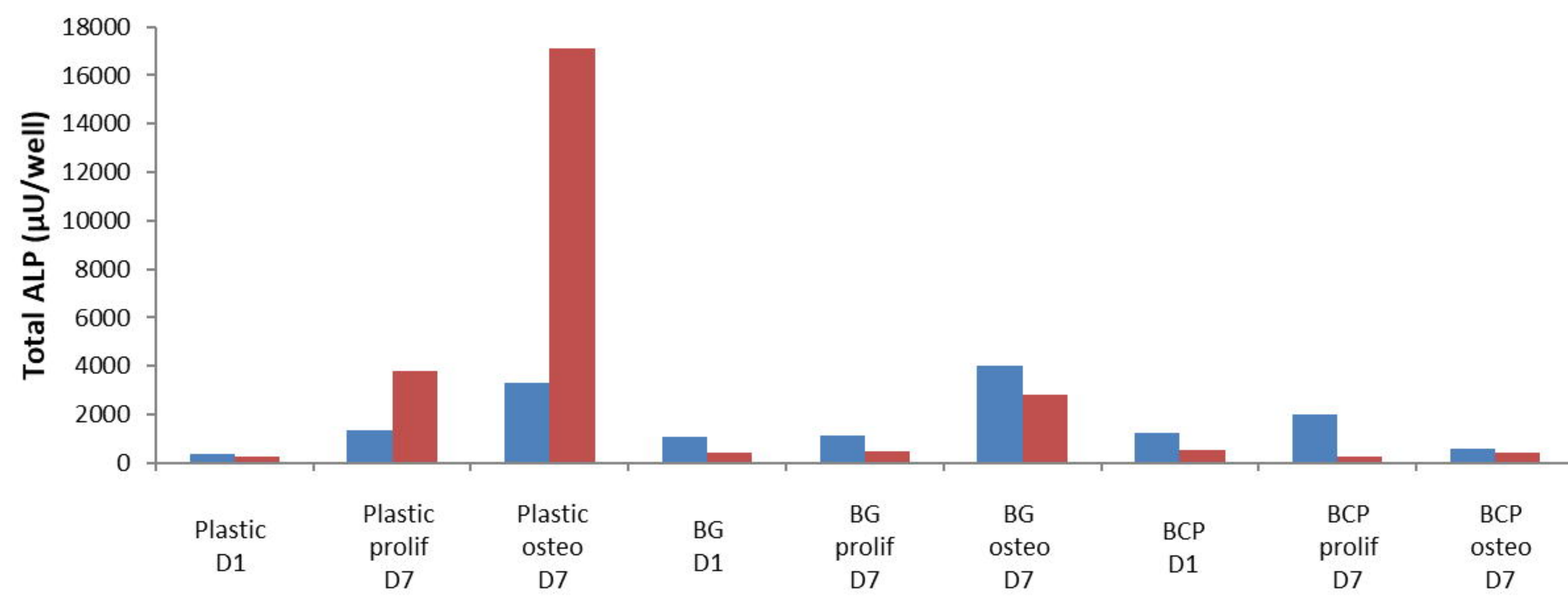

C

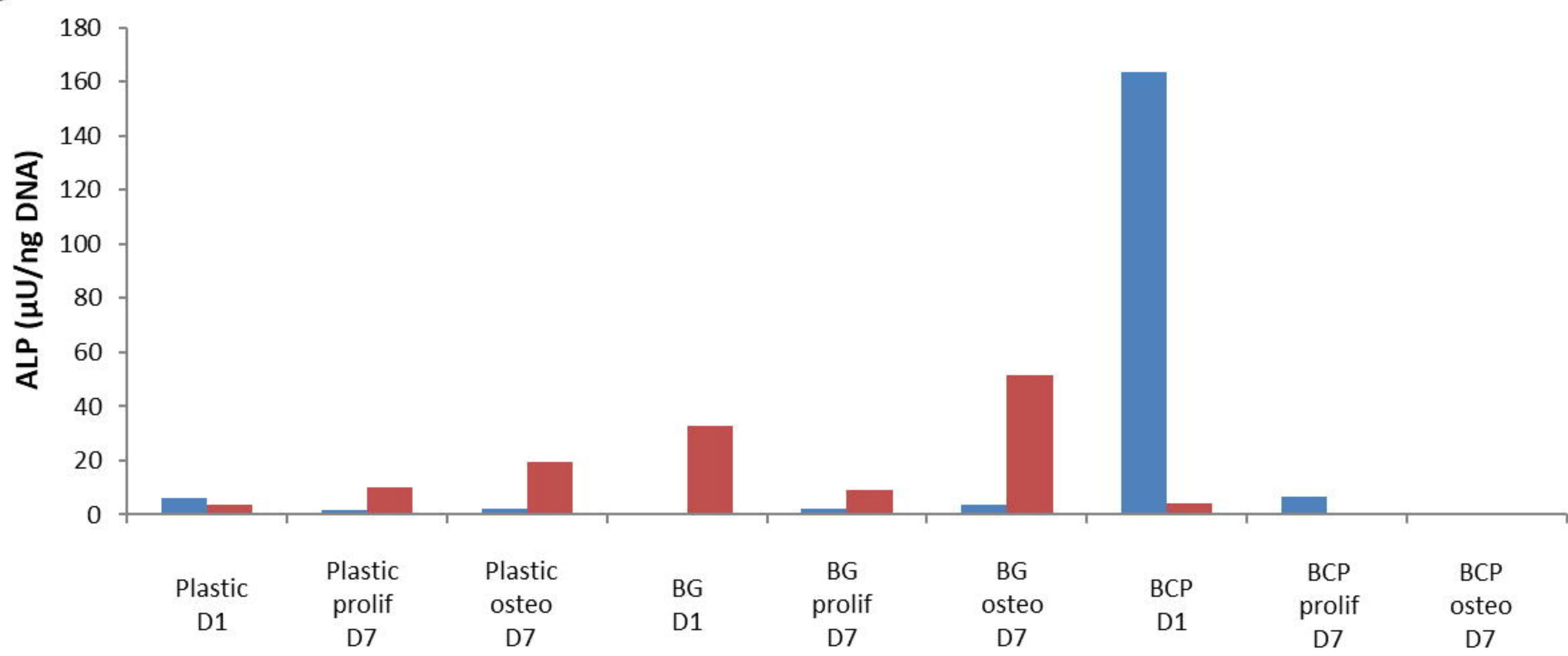


